IJMMS 30:11 (2002) 667-696

PII. S016117120200769X

http://ijmms.hindawi.com

(c) Hindawi Publishing Corp.

\title{
THE DE RHAM THEOREM FOR THE NONCOMMUTATIVE COMPLEX OF CENKL AND PORTER
}

\author{
LUIS FERNANDO MEJIAS
}

Received 22 May 2001

\begin{abstract}
We use noncommutative differential forms (which were first introduced by Connes) to construct a noncommutative version of the complex of Cenkl and Porter $\Omega^{*, *}(X)$ for a simplicial set $X$. The algebra $\Omega^{*, *}(X)$ is a differential graded algebra with a filtration $\Omega^{*, q}(X) \subset \Omega^{*, q+1}(X)$, such that $\Omega^{*, q}(X)$ is a $\mathbb{Q}_{q}$-module, where $\mathbb{Q}_{0}=\mathbb{Q}_{1}=\mathbb{Z}$ and $\mathbb{Q}_{q}=$ $\mathbb{Z}[1 / 2, \ldots, 1 / q]$ for $q>1$. Then we use noncommutative versions of the Poincaré lemma and Stokes' theorem to prove the noncommutative tame de Rham theorem: if $X$ is a simplicial set of finite type, then for each $q \geq 1$ and any $\mathbb{Q}_{q}$-module $M$, integration of forms induces a natural isomorphism of $\mathbb{Q}_{q}$-modules $I: H^{i}\left(\Omega^{*, q}(X), M\right) \rightarrow H^{i}(X ; M)$ for all $i \geq 0$. Next, we introduce a complex of noncommutative tame de Rham currents $\Omega_{*, *}(X)$ and we prove the noncommutative tame de Rham theorem for homology: if $X$ is a simplicial set of finite type, then for each $q \geq 1$ and any $\mathbb{Q}_{q}$-module $M$, there is a natural isomorphism of $\mathbb{Q}_{q^{-}}$ modules $I: H_{i}(X ; M) \rightarrow H_{i}\left(\Omega_{*, q}(X), M\right)$ for all $i \geq 0$.
\end{abstract}

2000 Mathematics Subject Classification: 55N35.

1. Introduction. Sullivan [22] used the de Rham complex $\Omega^{*}(X, \mathbb{Q})$ for a simplicial space $X$ of Whitney [23], to construct a free algebra model $M(X)$ of $\Omega^{*}$ such that the rational homotopy of $X$ could be computed. In an effort to use the idea of a "model" to compute homotopy group Miller [18] and Cartan [2] constructed a filtration $\mathscr{A}^{*}(X, \mathbb{Z})$ of the de Rham complex of polynomial forms $\mathscr{A}^{*}(X, \mathbb{Q})$ such that the cohomology $H^{i}\left(\mathscr{A}^{*, q}(X ; \mathbb{Z})\right)$ is isomorphic to the singular cohomology $H^{i}(X ; \mathbb{Z})$ for $i \leq q$. It turned out that a free model for $\mathscr{A}^{*}$ could not be constructed.

Cenkl and Porter $[6,7]$ constructed the so-called tame de Rham complex of polynomial forms $T^{*}(X, \mathbb{Z})$ with filtration $T^{*, q}(X) \subset T^{*, q+1}(X)$, depending on the degree of the polynomials and the forms, such that $T^{*, q}(X)$ is a $\mathbb{Q}_{q}$-module $\left(\mathbb{Q}_{0}=\mathbb{Q}_{1}=\right.$ $\mathbb{Z}$ and $\mathbb{Q}_{q}=\mathbb{Z}[1 / 2, \ldots, 1 / q]$ for $\left.q>1\right)$. They proved that there exists an isomorphism $I: H^{i}\left(T^{*, q}\left(X ; \mathbb{Q}_{q}\right)\right) \rightarrow H^{i}\left(X ; \mathbb{Q}_{q}\right)$ which is induced by integration of forms for all $q \geq 1$ and for all $i$. They also constructed a free model $T M(X)$ for $T(X, \mathbb{Z})$ which computes most of the torsion in the homotopy groups of $X$. However, the Steenrod operations cannot be introduced on $T M(X)$ (with proper localization).

The proof of Cenkl and Porter of the de Rham theorem for $T^{*}(X, \mathbb{Z})$ was done for a finite simplicial complex. This was later extended for a simplicial set by Boullay et al. [1]. They also dualized the situation and proved a homology version of the tame de Rham theorem [20]. Several attempts were made to build free models for spaces with $\mathbb{Z}_{p}$-coefficients (see $[10,13,19]$ ). 
Trying to construct a model for a space (along the lines of Sullivan, etc.) that would have cohomology operations, Karoubi [11] proposed enlarging the de Rham complex of commutative forms by considering noncommutative algebras (along the lines of Connes). Karoubi defined a noncommutative de Rham complex $\Omega(X)$ and proved the noncommutative de Rham theorem for a simplicial space $X$. A slightly more general version of the noncommutative de Rham theorem was proved by Cenkl in [4, 6]. Both proofs are functorial and in principle are based on the idea of Cartan. Some Steenrod operations are induced.

In this paper, we present another proof of the noncommutative de Rham theorem for a simplicial set of finite type. This proof is in the spirit of the classical de Rham theorem, that is, using integration. The possibility of such a proof (over a ring containing $\mathbb{Q}$ ) was suggested by Karoubi in [12]. However, we give a stronger result by defining a noncommutative tame de Rham complex $\Omega^{*, *}(X)$ (a noncommutative version of the de Rham complex of Cenkl and Porter).

THEOREM 1.1. Let $X$ be a simplicial set of finite type. Then for each $q \geq 1$ and any $\mathbb{Q}_{q}$-module $M$, there is a natural isomorphism of $\mathbb{Q}_{q}$-modules

$$
H^{i}\left(\Omega^{*, q}(X), M\right) \stackrel{\cong}{\longrightarrow} H^{i}(X ; M) \quad \forall i \geq 0 .
$$

The isomorphism is induced by integration.

Motivated by the work of Scheerer et al. [20], we also introduce a complex of noncommutative tame currents $\Omega_{*, *}(X)$ (dual of noncommutative tame forms) and prove the noncommutative tame de Rham theorem for homology.

THEOREM 1.2. Let $X$ be a simplicial set of finite type. Then for each $q \geq 1$ and any $\mathbb{Q}_{q}$-module $M$, there is a natural isomorphism of $\mathbb{Q}_{q}$-modules

$$
H_{i}(X ; M) \stackrel{\cong}{\longrightarrow} H_{i}\left(\Omega_{*, q}(X), M\right) \quad \forall i \geq 0 .
$$

The isomorphism is induced by integration.

Finally, we introduce a noncommutative version of the complex of tame currents presented in [20] and compare it with the complex $\Omega_{*, *}(X)$.

2. Simplicial objects. In this section, we present a brief introduction to the concept of simplicial objects as well as some examples. For more details see $[15,16,17]$.

A simplicial set $X$ is a graded set indexed on the nonnegative integers together with the face operators $d_{i}: X_{k} \rightarrow X_{k-1}$ and the degeneracy operators $s_{i}: X_{k} \rightarrow X_{k+1}$, $0 \leq i \leq k$, which satisfy the following identities:

(i) $d_{i} d_{j+1}=d_{j} d_{i}$ if $i \leq j$,

(ii) $s_{i} s_{j}=s_{j+1} s_{i}$ if $i \leq j$,

(iii) $d_{i} s_{j}=s_{j-1} d_{i}$ if $i<j$,

(iv) $d_{j} s_{j}=$ identity $=d_{j+1} s_{j}$,

(v) $d_{i} s_{j}=s_{j} d_{i-1}$ if $i>j+1$.

The elements of $X_{k}$ are called $k$-simplices. Let $X$ and $Y$ be two simplicial sets. A simplicial map $f: X \rightarrow Y$ is a map of graded sets of degree zero which commutes with the face and degeneracy operators. 
If $X$ and $Y$ are two simplicial sets, the Cartesian product $X \times Y$ is the simplicial set with $(X \times Y)_{k}=X_{k} \times Y_{k}$ and

$$
d_{i}(x, y)=\left(d_{i} x, d_{i} y\right), \quad s_{i}(x, y)=\left(s_{i} x, s_{i} y\right), \quad \forall x \in X_{k}, y \in Y_{k}, 0 \leq i \leq k .
$$

EXAMPLE 2.1. Let $V$ be any partially ordered set. Let $X_{k}$ be the set of all finite sequences $\left(x_{0}, \ldots, x_{k}\right)$, with $x_{0} \leq \cdots \leq x_{k}, x_{0}, \ldots, x_{k} \in V$. Define $d_{i}: X_{k} \rightarrow X_{k-1}$ and $s_{i}: X_{k} \rightarrow X_{k+1}, 0 \leq i \leq k$, by

$$
\begin{aligned}
d_{i}\left(x_{0}, \ldots, x_{k}\right) & =\left(x_{0}, \ldots, x_{i-1}, \hat{x}_{i}, x_{i+1}, \ldots, x_{k}\right) \quad\left(\text { omit } x_{i}\right), \\
s_{i}\left(x_{0}, \ldots, x_{k}\right) & =\left(x_{0}, \ldots, x_{i-1}, x_{i}, x_{i}, x_{i+1}, \ldots, x_{k}\right) \quad\left(\text { double } x_{i}\right) .
\end{aligned}
$$

Then $X=\left\{X_{k}\right\}$ is a simplicial set.

EXAMPLE 2.2. Let $\Delta$ denote the category whose objects are all finite sequences of integers $\Delta(n)=\{0,1, \ldots, n\}$ and the morphisms are all the increasing functions $f: \Delta(n) \rightarrow \Delta(m)$ (for all $0 \leq i \leq j \leq n$, we have $f(i) \leq f(j))$.

Define the morphisms $\delta_{i}: \Delta(n-1) \rightarrow \Delta(n)$ and $\sigma_{i}: \Delta(n+1) \rightarrow \Delta(n)$, for $0 \leq i \leq$ $n$, by

$$
\delta_{i}(j)=\left\{\begin{array}{ll}
j & \text { if } j<i, \\
j+1 & \text { if } j \geq i,
\end{array} \quad \sigma_{i}(j)= \begin{cases}j & \text { if } j \leq i, \\
j-1 & \text { if } j>i .\end{cases}\right.
$$

Then every $f \in \operatorname{Hom}(\Delta(n), \Delta(m))$ can be written as the product of finitely many $\delta$ 's and $\sigma$ 's.

A simplicial object in a category $\mathscr{C}$ is a contravariant functor $F: \Delta \rightarrow \mathscr{C}$. A simplicial set $X$ can be identified with a simplicial object $X$ in the category of sets Set, $F: \Delta \rightarrow$ Set, $X=F\left(\Delta_{n}\right)=X$ (see [15, page 233] or [17, page 4]).

A simplicial $\Lambda$-module is a simplicial object in the category of $\Lambda$-modules Mod. If $M$ and $N$ are simplicial $\Lambda$-modules, then the tensor product $M \otimes N$ is a simplicial $\Lambda$-module. The face and degeneracy operators $d_{i}$ and $s_{i}$ on $(M \otimes N)_{k}=M_{k} \otimes N_{k}$ are given by

$$
d_{i}(x \otimes y)=d_{i} x \otimes d_{i} y, \quad s_{i}(x \otimes y)=s_{i} x \otimes s_{i} y, \quad \forall x \in X_{k}, y \in Y_{k}, 0 \leq i \leq k .
$$

A simplicial graded algebra $\mathscr{A}^{*}=\oplus_{n \geq 0} \mathscr{A}^{n}$ is a family of graded algebras $\mathscr{A}_{k}^{*}=$ $\oplus_{n \geq 0} \mathscr{A}_{k}^{n}, k=0,1,2, \ldots$, over a commutative ring $\Lambda$ which is a simplicial set and the face and degeneracy operators $d_{i}$ and $s_{i}$ are morphisms of graded algebras.

EXAMPLE 2.3. Let $\Delta_{n}=\left\{\left(a_{0}, \ldots, a_{n}\right) \in \mathbb{R}^{n+1} \mid 0 \leq a_{i} \leq 1, \sum a_{i}=1\right\}$ be the standard $n$-simplex (Figure 2.1). The maps $\delta_{i}: \Delta_{n-1} \rightarrow \Delta_{n}$ and $\sigma_{i}: \Delta_{n+1} \rightarrow \Delta_{n}$ are defined by

$$
\begin{aligned}
& \delta_{i}\left(x_{0}, \ldots, x_{n-1}\right)=\left(x_{0}, \ldots, x_{i-1}, 0, x_{i}, \ldots, x_{n-1}\right), \\
& \sigma_{i}\left(x_{0}, \ldots, x_{n+1}\right)=\left(x_{0}, \ldots, x_{i-1}, x_{i}+x_{i+1}, x_{i+2}, \ldots, x_{n+1}\right) .
\end{aligned}
$$

Let $\mathscr{P}_{n}$ be the collection of the polynomials $f: \Delta_{n} \rightarrow \mathbb{R}$ with $\mathbb{Z}$-coefficients and let $\mathscr{P}=\left\{\mathscr{P}_{n}\right\}_{n \geq 0}$. Then $\mathscr{P}$ is a simplicial set. The face and degeneracy maps are the maps $\partial_{i}: \mathscr{P}_{n} \rightarrow \mathscr{P}_{n-1}$ and $s_{i}: \mathscr{P}_{n} \rightarrow \mathscr{P}_{n+1}$ defined, for each $f \in \mathscr{P}_{n}$, by

$$
\partial_{i}(f)=f \circ \delta_{i}, \quad s_{i}(f)=f \circ \sigma_{i},
$$



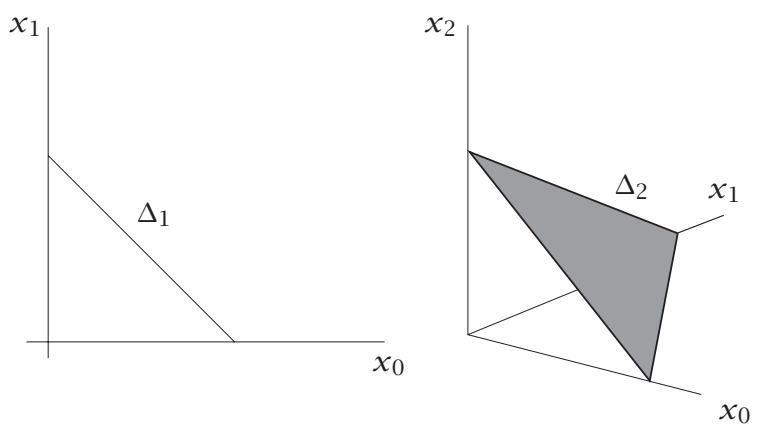

FIGURE 2.1. The standard simplexes $\Delta_{1}$ and $\Delta_{2}$.

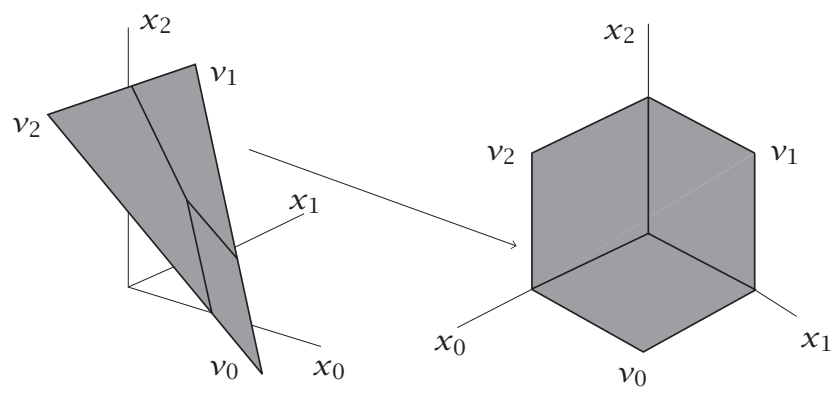

FIGURE 2.2. The 2-simplexes $\Delta_{2}$.

$\left(\partial_{i}\right.$ and $s_{i}$ are the pullbacks of $\delta_{i}$ and $\sigma_{i}$ ). Multiplication of polynomials induces an algebra structure on $\mathscr{P}$. Then $\mathscr{P}$ is a simplicial algebra.

EXAMPLE 2.4. Instead of the standard $n$-simplex $\Delta_{n}$ as in Example 2.3, we consider $\Delta_{n}$ to be the subset of the boundary of $I^{n+1}$ (the standard $(n+1)$-cube in $\mathbb{R}^{n+l}$ ) given by

$$
\left\{\left(x_{0}, \ldots, x_{n}\right) \in \mathbb{R}^{n+l}: 0 \leq x_{i} \leq 1, \prod_{i} x_{i}=0\right\}
$$

that is, $\Delta_{n}$ is identified with the backfaces of $I^{n+1}$ (Figure 2.2).

Define the maps $\delta_{i}: \Delta_{n-1} \rightarrow \Delta_{n}$ and $\sigma_{i}: \Delta_{n+1} \rightarrow \Delta_{n}$ by

$$
\begin{aligned}
& \delta_{i}\left(x_{0}, \ldots, x_{n-1}\right)=\left(x_{0}, \ldots, x_{i-1}, 1, x_{i}, x_{i+1}, \ldots, x_{n-1}\right), \\
& \sigma_{i}\left(x_{0}, \ldots, x_{n+1}\right)=\left(x_{0}, \ldots, x_{i-1}, x_{i} \cdot x_{i+1}, x_{i+2}, \ldots, x_{n+1}\right)
\end{aligned}
$$

(see $[5,7])$. A $k$-face $F$ of $\Delta_{n}$ is determined for two disjoint sets $A=\left\{a_{1}, a_{2}, \ldots, a_{k+1}\right\}$ and $B=\left\{b_{1}, b_{2}, \ldots, b_{n-k}\right\}$ such that $0 \leq a_{1}<a_{2}<\cdots<a_{k+1} \leq n, 0 \leq b_{1}<b_{2}<$ $\cdots<b_{n-k} \leq n, A \cup B=\{0,1, \ldots, n\}, 0 \leq x_{i} \leq 1, \prod_{i \in A} x_{i}=0$, and $x_{j} \equiv 1$ for all $j \in B$. Sometimes we use the notation $F=F(A, B)$. Figure 2.3 shows the 1 -face $F(A, B)$ of the 2-simplex $\Delta_{2}$ for $A=\{1,2\}$ and $B=\{0\}$. 


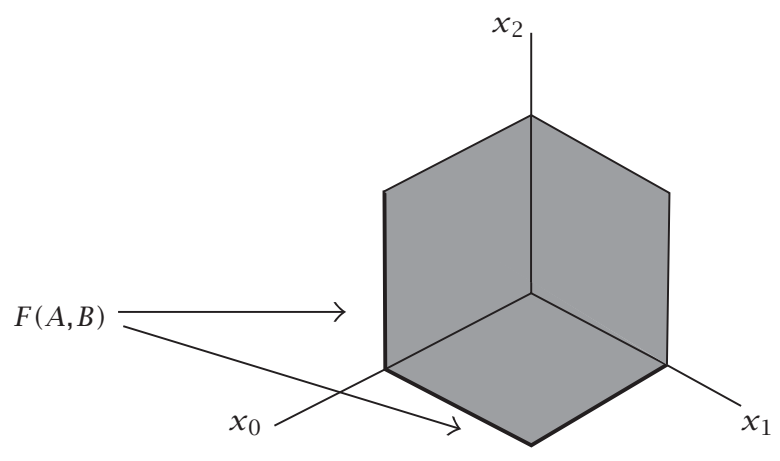

FIGURE 2.3. A 1-face of $\Delta_{2}$.

Let $\mathscr{T}_{n}$ be the collection of the polynomials $f: \Delta_{n} \rightarrow \mathbb{R}$ with $\mathbb{Z}$-coefficients and let $\mathscr{T}=\left\{\mathscr{T}_{n}\right\}_{n \geq 0}$. Then $\mathscr{T}$ is a simplicial algebra. The face and degeneracy maps are the maps $\partial_{i}: \mathscr{P}_{n} \rightarrow \mathscr{P}_{n-1}$ and $s_{i}: \mathscr{P}_{n} \rightarrow \mathscr{P}_{n+1}$ defined, for each $f \in \mathscr{P}_{n}$, by

$$
\partial_{i}(f)=f \circ \delta_{i}, \quad s_{i}(f)=f \circ \sigma_{i},
$$

$\left(\partial_{i}\right.$ and $s_{i}$ are the pullbacks of $\delta_{i}$ and $\sigma_{i}$ ).

EXAMPLE 2.5. Let $\Phi_{n}$ be the ideal generated by the polynomial $\prod_{j=0}^{n} x_{i}$. Then $\mathscr{P}_{n}$ can be identified with the quotient $\mathscr{T}_{n}=\mathbb{Z}\left[x_{0}, \ldots, x_{n}\right] / \mathscr{I}_{n}$. Let $\mathscr{T}=\left\{\mathscr{T}_{n}\right\}_{n \geq 0}$. Multiplication on $\mathbb{Z}\left[x_{0}, \ldots, x_{n}\right]$ induces structure of $\mathbb{Z}$-algebra on $\mathscr{T}_{n}$. Then $\mathscr{T}$ is a simplicial algebra.

Let $X$ be a simplicial set and let $C_{n}(X)$ be the free group on $X_{n}$. Denote by $C_{*}(X)$ the chain complex $\left(C_{n}(X), \partial\right)$ with the boundary operator $\partial=\sum_{i=0}^{n}(-1)^{i} d_{i}$. Elements of $C_{n}(X)$ are called $n$-chains in $X$. If $X$ is a simplicial set and $G$ is an abelian group, then the homology of $X$ with coefficients in $G$ is defined by

$$
H_{*}(X ; G)=H\left(C_{*}(X) \otimes G\right) .
$$

Denote by $C^{*}(X)$ the complex $\left(C^{n}(X), \delta\right)$ of cochains in $X$ with coefficients in $G$ where $C^{n}(X ; G)=\operatorname{Hom}\left(C_{n}(X), G\right)$ and the coboundary operator $\delta$ is the dual of $\partial$. The cohomology of $X$ with coefficients in $G$ is defined by

$$
H^{*}(X ; G)=H\left(C^{*}(X), G\right)
$$

3. The complex of Cenkl-Porter. Cenkl and Porter [7] first proved the de Rham theorem for the complex of cubical differential forms for a space of finite type using integration. Later Boullay, Kiefer, Majewski, Stelzer, Scheerer, Unsöld, and Vogt [1] introduced the complex of Cenkl and Porter or the de Rham complex of tame differential forms and proved the de Rham theorem for a simplicial set following Cartan's ideas.

In this section, we present the complex of Cenkl and Porter which is the complex of compatible differential forms on the backfaces of the standard cube and state the tame de Rham theorem. 
Let $\Delta_{n} \subset \mathbb{R}^{n+1}$ denote the standard simplex (Example 2.4). A basic form of weight $q$ on $\Delta_{n}$ in the coordinates $x_{0}, x_{1}, \ldots, x_{n}$ is a differential form

$$
x_{i_{1}}^{a_{1}} \cdots x_{i_{j}}^{a_{j}} x_{k_{1}}^{b_{1}} d x_{k_{1}} \wedge \cdots \wedge x_{k_{p}}^{b_{p}} d x_{k_{p}}
$$

where $\left\{i_{1}, \ldots, i_{j}\right\}$ and $\left\{k_{1}, \ldots, k_{p}\right\}$ are disjoint subsets of $\{0,1, \ldots, n\}$, the $a$ 's and $b$ 's are nonnegative integers, and $q=\max \left\{a_{1}, \ldots, a_{j}, b_{1}+1, \ldots, b_{p}+1\right\}$. Let $\mathbb{Q}_{q}=\mathbb{Z}[1 / 2, \ldots, 1 / q]$ be the smallest subring of the rationals such that $Q_{q}$ contains $1 / p$ if $0<p \leq q$ for $q>1$, and $\mathbb{Q}_{0}=\mathbb{Q}_{1}=\mathbb{Z}$. Denote by $T^{p, q}\left(\Delta_{n}\right)$ the module of $\mathbb{Q}_{q}$-linear combinations of basic $p$-forms of weight less than or equal to $q$. The wedge product $\wedge$ extends to a map

$$
\wedge: T_{n}^{p_{1}, q_{1}}(\mathbb{Z}) \otimes T_{n}^{p_{2}, q_{2}}(\mathbb{Z}) \longrightarrow T_{n}^{p_{1}+p_{2}, q_{1}+q_{2}}(\mathbb{Z})
$$

and the usual differential $d$ extends to a morphism of $\mathbb{Z}$-modules $d: T_{n}^{p, q}(\mathbb{Z}) \rightarrow$ $T_{n}^{p+1, q}(\mathbb{Z})$. We also have the inclusion map $T_{n}^{p, q} \mathbb{Z} \hookrightarrow T_{n}^{p, q+1}(\mathbb{Z})$. Then for every $n \geq 0$, $T^{*, *}=\left\{T^{p, q}\left(\Delta_{n}\right)\right\}_{n \geq 0}$ is a simplicial differential graded algebra (DGA) with filtration. For the proofs of the next two results we refer to $[1,7]$.

Proposition 3.1. If $\Delta_{p}$ is a p-simplex contained in $\Delta_{n}$, and $\omega \in T^{p, q}\left(\Delta_{n}\right)$, then

$$
\int_{\Delta_{p}} \omega \in \mathbb{Q}_{q}
$$

Let $X=\left\{X_{n}\right\}$ be a simplicial set and let $T(X)=\operatorname{Mor}\left(T^{*, *}, X\right)$ (morphisms of simplicial sets). The Stokes' theorem implies that for any $q \geq 0$, integration of tame forms induces a map of cochain complexes $I: T^{*, q}(X) \rightarrow C^{*}\left(X ; \mathbb{Q}_{q}\right)$. Then we have the following theorem.

THEOREM 3.2 (the tame de Rham theorem). Let $X$ be a simplicial set of finite type. Then for each $q \geq 1$ and any $Q_{q}$-module $M$ there is a natural isomorphism of $Q_{q}$ modules

$$
H^{i}\left(T^{*, q}(X), M\right) \stackrel{\cong}{\longrightarrow} H^{i}(X ; M) \quad \forall i \geq 0 .
$$

The isomorphism is induced by integration.

4. Noncommutative differential forms. In this section, we present the complex of noncommutative differential forms or the noncommutative de Rham complex, which is a generalization of the standard de Rham complex on a manifold $M$, but the algebra of smooth functions on $M$ is replaced by an arbitrary associative algebra with unit. Noncommutative forms were introduced by Connes [8, 9]. Karoubi [11] used noncommutative forms to define the noncommutative de Rham complex $\Omega(X)$ and proved a noncommutative version of the de Rham theorem for a simplicial space $X$ [12]. Here we present the basic properties of the noncommutative de Rham complex of an algebra $\mathscr{A}$ over a commutative ring $\Lambda$.

Let $\mathscr{A}$ be an algebra over a commutative ring $\Lambda$ (with unit). Let $\mu: \mathscr{A} \otimes \mathscr{A} \rightarrow \mathscr{A}$ denote the multiplication operation on $\mathscr{A}$ (all rings are considered to be commutative and 
unitary, and all algebras are with unit). The differential forms of degree $n$ are the elements of the tensor product of $\Lambda$-algebras

$$
\mathscr{T}^{n}(\mathscr{A})=\underbrace{\mathscr{A} \otimes_{\Lambda} \mathscr{A} \otimes_{\Lambda} \cdots \otimes_{\Lambda} \mathscr{A}}_{n+1 \text { times }} .
$$

The algebra $\mathscr{T}^{*}(\mathscr{A})=\otimes_{n \geq 0} T^{n}(\mathscr{A})$ is a $\Lambda$-algebra with the multiplication $\cdot: \mathscr{T}^{n}(\mathscr{A}) \otimes$ $\mathscr{T}^{m}(\mathscr{A}) \rightarrow \mathscr{T}^{n+m}(A)$ defined by the formula

$$
\left(a_{0} \otimes a_{1} \otimes \cdots \otimes a_{n}\right) \cdot\left(b_{0} \otimes b_{1} \otimes \cdots \otimes b_{m}\right)=a_{0} \otimes a_{1} \otimes \cdots \otimes\left(a_{n} \cdot b_{0}\right) \otimes b_{1} \otimes \cdots \otimes b_{m} .
$$

The differential operator $D: \mathscr{T}^{n}(\mathscr{A}) \rightarrow \mathscr{T}^{n+1}(\mathscr{A})$ is defined by the formula

$$
\begin{aligned}
D\left(a_{0} \otimes a_{1} \otimes \cdots \otimes a_{n}\right)= & 1 \otimes a_{0} \otimes a_{1} \otimes \cdots \otimes a_{n} \\
& +\sum_{j=1}^{n}(-1)^{j} a_{0} \otimes a_{1} \otimes \cdots \otimes a_{j-1} \otimes 1 \otimes a_{j} \cdots a_{n} \\
& +(-1)^{n+1} a_{0} \otimes a_{1} \otimes \cdots \otimes a_{n} \otimes 1 .
\end{aligned}
$$

THEOREM 4.1. If $\omega \in \mathscr{T}^{n}(\mathscr{A})$ and $\theta \in \mathscr{T}^{m}(\mathscr{A})$, then

(1) $D^{2}(\omega)=0$;

(2) $D(\omega \cdot \theta)=D(\omega) \cdot \theta+(-1)^{n} \omega \cdot D(\theta)$ (Leibniz identity).

Then $\mathscr{T}^{*}(\mathscr{A})$ is a DGA and the cohomology of the complex $\left(\mathscr{T}^{*}(\mathscr{A}), D\right)$ is trivial. Suppose that $\mathscr{A}$ is an augmented $\Lambda$-algebra with an augmentation $\lambda: \mathscr{A} \rightarrow \Lambda$ (morphism of rings) such that $\lambda(1)=1$. Consider the map of modules $\imath_{\lambda}: \mathscr{T}^{n}(\mathscr{A}) \rightarrow \mathscr{T}^{n-1}(\mathscr{A})$ defined by

$$
\imath_{\lambda}\left(a_{0} \otimes \cdots \otimes a_{n}\right)=\lambda\left(a_{0}\right)\left(a_{1} \otimes \cdots \otimes a_{n}\right) .
$$

THEOREM 4.2. Let $\mathscr{A}$ be an augmented $\Lambda$-algebra with an augmentation $\lambda: \mathscr{A} \rightarrow \Lambda$ such that $\lambda(1)=1$. The map $\imath_{\lambda}: \mathscr{T}^{n}(\mathscr{A}) \rightarrow \mathscr{T}^{n-1}(\mathscr{A})$ is a contracting homotopy,

$$
D \imath_{\lambda}+\imath_{\lambda} D=1 .
$$

Define $\Omega^{0}(\mathscr{A})=\mathscr{A}$ and $\Omega^{1}(\mathscr{A})=\operatorname{ker} \mu$, the $\Lambda$-module $\Omega^{1}(\mathscr{A})$ is an $\mathscr{A}$-bimodule. The noncommutative differential forms of degree $n$ are the elements of the tensor product of $A$-modules

$$
\Omega^{n}(\mathscr{A})=\underbrace{\Omega^{1}(\mathscr{A}) \otimes_{\mathscr{A}} \Omega^{1}(\mathscr{A}) \otimes_{\mathscr{A}} \cdots \otimes_{\mathscr{A}} \Omega^{1}(\mathscr{A})}_{n \text { times }} .
$$

The product of differential forms is defined by juxtaposition of tensor products. Then the direct sum

$$
\Omega^{*}(\mathscr{A})=\bigoplus_{n \geq 0} \Omega^{n}(\mathscr{A})
$$

is a graded algebra. The differential $d: \Omega^{0}(\mathscr{A}) \rightarrow \Omega^{1}(\mathscr{A})$ is defined by the formula

$$
d(a)=1 \otimes a-a \otimes 1
$$


Thus we have the isomorphism of $\Lambda$-modules $\mathscr{A} \otimes \mathscr{A} / \Lambda \rightarrow \Omega^{1}(\mathscr{A})$ such that $a \otimes \bar{b} \mapsto$ $a d b$. Then $\Omega^{n}(\mathscr{A})$ can be identified with the tensor product of $\Lambda$-modules

$$
\mathscr{A} \otimes \underbrace{\mathscr{A} / \Lambda \otimes \mathscr{A} / \Lambda \otimes \cdots \mathscr{A} / \Lambda}_{n \text { times }} .
$$

A noncommutative differential form of degree $n$ can be written as a linear combination of terms of the form $a_{0} d a_{1} d a_{2} \cdots d a_{n}$ and the morphism $d$ extends to forms of degree $n$ of $\Omega^{n}(\mathscr{A})$ by the formula

$$
d\left(a_{0} d a_{1} \cdots d a_{n}\right)=d a_{0} d a_{1} \cdots d a_{n}=1 d a_{0} d a_{1} \cdots d a_{n}
$$

THEOREM 4.3. If $\omega \in \Omega^{n}(\mathscr{A})$ and $\theta \in \Omega^{m}(\mathscr{A})$, then

(1) $d^{2}(\omega)=0$;

(2) $d(\omega \cdot \theta)=d(\omega) \cdot \theta+(-1)^{n} \omega \cdot d(\theta)$ (Leibniz identity).

REMARK 4.4. The DGA $\Omega^{*}(\mathscr{A})$ is called the differential enveloping of $\mathscr{A}$, and it is the solution of a universal problem: for a DGA $\mathscr{B}^{*}$ and an algebra morphism $f: \mathscr{A} \rightarrow \mathscr{S}^{0}$, there exists a unique morphism of DGA's $f^{*}: \Omega^{*}(\mathscr{A}) \rightarrow \mathscr{B}^{*}$ which agrees with $f$ at degree 0 . The complex $\left(\Omega^{*}(\mathscr{A}), d\right)$ is known as the universal differential calculus of $\mathscr{A}$ or as the noncommutative de Rham complex of $\mathscr{A}$.

There is an inclusion of DGA's sending $\Omega^{*}(\mathscr{A})$ to $\mathscr{T}^{*}(\mathscr{A})$. On the other hand, for any $n \geq 0$ there is a projection operator $J: \mathscr{T}^{n}(\mathscr{A}) \rightarrow \Omega^{n}(\mathscr{A})$ defined by $J\left(a_{0} \otimes a_{1} \otimes \cdots \otimes\right.$ $\left.a_{n}\right)=a_{0} d a_{1} \cdots d a_{n}$.

A noncommutative differential form $\omega$ is said to be closed if $d \omega=0$. We say that $\omega \in \Omega^{n}(\mathscr{A})$ is exact if there exists $\eta \in \Omega^{n-1}(\mathscr{A})$ such that $\omega=d \eta$. The fact that the complex $\left(\Omega^{*}(\mathscr{A}), d\right)$ has trivial cohomology is known as the noncommutative Poincaré lemma.

LEMMA 4.5. Let $\mathscr{A}$ be an augmented $\Lambda$-algebra, then every closed form $\omega \in \Omega^{*}(\mathscr{A})$ is exact.

Proof. As in Theorem 4.2, let $\lambda: \mathscr{A} \rightarrow \Lambda$ be a $\Lambda$-linear form with $\lambda(1)=1$. We prove that there exists a homotopy contraction $j_{\lambda}: \Omega^{n}(\mathscr{A}) \rightarrow \Omega^{n-1}(\mathscr{A})$. To define $J_{\lambda}$ we express elements of $\Omega^{n}(\mathscr{A})$ as elements of $\mathscr{T}^{n+1}(\mathscr{A})$ using inclusion, next we apply $\imath_{\lambda}$, and then we apply the projection $J$. Thus for $\omega=a_{0} d a_{1} \cdots d a_{n}$, we get $J_{\lambda}(\omega)=\lambda\left(a_{0}\right) a_{1} d a_{2} \cdots d a_{n}-\lambda\left(a_{0} a_{1}\right) d a_{2} \cdots d a_{n}$. First we show that $\iota_{\lambda}$ is well defined. Obviously it is enough to prove that $t_{\lambda}: \Omega^{1}(\mathscr{A}) \rightarrow \Omega^{0}(\mathscr{A})$ is well defined. Let $\omega=a_{0} d a_{1} \in \Omega^{1}(\mathscr{A}), a \in \Lambda$, and $a_{1}^{\prime}=a_{1}+a \cdot 1 \in \mathscr{A}$. Then $j_{\lambda}\left(a_{0} d a_{1}^{\prime}\right)=\lambda\left(a_{0}\right) a_{1}^{\prime}-$ $\lambda\left(a_{0} a_{1}^{\prime}\right) \cdot 1=\lambda\left(a_{0}\right) a_{1}-\lambda\left(a_{0} a_{1}\right) \cdot 1=J_{\lambda}\left(a_{0} d a_{1}\right)$. Now for $\omega=a_{0} d a_{1} \cdots d a_{n} \in \Omega^{n}(\mathscr{A})$, we have

$$
\begin{aligned}
& d J_{\lambda}(\omega)+j_{\lambda} d(\omega)=\lambda\left(a_{0}\right) d a_{1} d a_{2} \cdots d a_{n}-\lambda\left(a_{0} a_{1}\right) d 1 d a_{2} \cdots d a_{n} \\
& +\lambda(1) a_{0} d a_{1} d a_{2} \cdots d a_{n}-\lambda\left(1 \cdot a_{0}\right) d a_{1} \cdots d a_{n} \\
& =w \text {. }
\end{aligned}
$$

But $d \omega=0$. Therefore $d_{J_{\lambda}}(\omega)=\omega$. 
If $\mathscr{A}=\left\{\mathscr{A}_{n}\right\}_{n \geq 0}$ is a simplicial algebra, then $\Omega^{*}(\mathscr{A})=\left\{\Omega^{*}\left(\mathscr{A}_{n}\right)\right\}_{n \geq 0}$ is a simplicial DGA. Next we define the face and degeneracy operators for $\Omega^{*}(\mathscr{A})$.

Let $\mathscr{A}=\left\{\mathscr{A}_{n}^{*}\right\}_{n \geq 0}$ be a simplicial graded algebra. For each $n$ consider the simplicial tensor algebra

$$
\mathscr{T}^{*}\left(\mathscr{A}_{n}\right)=\bigoplus_{p \geq 0} \mathscr{A}_{n}^{\otimes p}
$$

where the face and degeneracy operators $\partial_{i}: \mathscr{A}_{n}^{\otimes p} \rightarrow \mathscr{A}_{n-1}^{\otimes p}$ and $s_{i}: \mathscr{A}_{n}^{\otimes p} \rightarrow \mathscr{A}_{n+1}^{\otimes p}$ are defined by

$$
\begin{aligned}
& \partial_{i}\left(a_{0} \otimes a_{1} \otimes \cdots \otimes a_{p}\right)=\partial_{i} a_{0} \otimes \partial_{i} a_{1} \otimes \cdots \otimes \partial_{i} a_{p}, \\
& s_{i}\left(a_{0} \otimes a_{1} \otimes \cdots \otimes a_{p}\right)=s_{i} a_{0} \otimes s_{i} a_{1} \otimes \cdots \otimes s_{i} a_{p} .
\end{aligned}
$$

Proposition 4.6. Let $\mathscr{A}=\left\{\mathscr{A}_{n}^{*}\right\}_{n \geq 0}$ be a simplicial algebra. If $D$ is the differential on $\mathscr{T}^{*}\left(\mathscr{A}_{n}\right)$, then $\mathscr{T}^{*}(\mathscr{A})$ is a simplicial DGA.

Observe that the restriction of $\partial_{i}$ to $\Omega^{1}\left(\mathscr{A}_{n}\right)$ applied to $a d b \in \Omega^{1}\left(\mathscr{A}_{n}\right)$ is

$$
\begin{aligned}
\partial_{i}(a d b) & =\partial_{i}(a \otimes b-a b \otimes 1)=\partial_{i}(a) \otimes \partial_{i}(b)-\partial_{i}(a b) \otimes 1 \\
& =\partial_{i}(a) \otimes \partial_{i}(b)-\partial_{i}(a) \partial_{i}(b) \otimes 1 \in \mathscr{A}_{n-1} \otimes \mathscr{A}_{n-1} .
\end{aligned}
$$

If $\mu_{n-1}$ denotes multiplication on $A_{n-1}$, then

$$
\begin{aligned}
\mu_{n-1}\left(\partial_{i}(a d b)\right) & =\mu_{n-1}\left(\partial_{i}(a) \otimes \partial_{i}(b)-\partial_{i}(a) \partial_{i}(b) \otimes 1\right) \\
& =\partial_{i}(a) \cdot \partial_{i}(b)-\partial_{i}(a) \partial_{i}(b) \cdot 1=0 .
\end{aligned}
$$

Therefore $a d b \in \operatorname{ker} \mu_{n-1}=\Omega^{1}\left(\mathscr{A}_{n-1}\right)$. In particular, if we take elements of the form $d a \in \Omega^{1}\left(A_{n-1}\right)$, then we get

$$
\partial_{i}(d a)=\partial_{i}(1 \otimes a-a \otimes 1)=1 \otimes \partial_{i} a-\partial_{i} a \otimes 1=d\left(\partial_{i} a\right) .
$$

Then extend $\partial_{i}$ to $\Omega^{P}(\mathscr{A})$ by setting

$$
\partial_{i}\left(a_{0} d a_{1} \cdots d a_{p}\right)=\partial_{i}\left(a_{0}\right) d\left(\partial_{i} a_{1}\right) \cdots d\left(\partial_{i} a_{p}\right) \in \Omega^{P}\left(A_{n-1}\right) .
$$

Similarly $s_{i}$ can be extended to $\Omega^{P}(\mathscr{A})$. Then we have the following proposition.

Proposition 4.7. If $\mathscr{A}=\left\{\mathscr{A}_{n}\right\}_{n \geq 0}$ is a simplicial graded algebra, then $\Omega^{*}(\mathscr{A})=$ $\left\{\Omega^{*}\left(A_{n}\right)\right\}_{n \geq 0}$ is a simplicial DGA.

A noncommutative version of the de Rham theorem was proved by Karoubi in [12]. Karoubi considered $\mathscr{A}_{n}$ to be the quotient $\Lambda$-algebra $\Lambda\left[x_{0}, x_{1}, \ldots, x_{n}\right] /\left(x_{0}+x_{1}+\right.$ $\left.\cdots+x_{n}-1\right)$. Let $\Omega^{*}\left(\mathscr{A}_{n}\right)$ be the algebra of noncommutative forms on $\mathscr{A}_{n}$ and let $\mathscr{A}=\left\{\mathscr{A}_{n}\right\}_{n \geq 0}$. The algebra $\Omega^{*}\left(\mathscr{A}_{n}\right)$ is the noncommutative algebra generated by the symbols $x_{i}$ and $d x_{i}, 0 \leq i \leq n$ and the following relations:

$$
\sum_{i=0}^{n} x_{i}=1, \quad \sum_{i=0}^{n} d x_{i}=0, \quad x_{i} x_{j}=x_{j} x_{i} .
$$

Then we have the following theorem. 
THEOREM 4.8 (the noncommutative de Rham theorem). Let $X$ be a simplicial set and let $\Omega^{*}(X)=\operatorname{Mor}\left(X, \Omega^{*}(\mathscr{A})\right)$. Then there exists a natural isomorphism of $\Lambda$-modules

$$
H^{i}\left(\Omega^{*}(x)\right) \cong H^{i}(X ; \Lambda) \quad \forall i \geq 0 .
$$

A slightly more general version of Theorem 4.8 was proved by Cenkl in [3, 4].

5. The noncommutative complex of Cenkl-Porter. In [12], Karoubi conjectured that the noncommutative de Rham theorem could be proved using integration of noncommutative differential forms assuming that $\Lambda$ is a ring containing the ring of the rational numbers $\mathbb{Q}$. We present a solution of a more general problem by considering a noncommutative version of the tame de Rham complex of Cenkl-Porter. This complex is constructed by defining a filtration on $\Omega^{*}(\mathscr{T})$, the algebra of noncommutative differential forms on $\mathscr{T}=\oplus_{n \geq 0} \mathscr{T}_{n}$, where $\mathscr{T}_{n}$ are the polynomials restricted to $n$-simplex $\Delta_{n}$ (see Example 2.4). Then we prove some basic properties of that complex. In particular we prove the noncommutative tame Poincaré lemma.

REMARK 5.1. Propositions 4.6 and 4.7 imply that $\Omega^{*}(\mathscr{T})$ is a simplicial DGA.

We establish some conventions of notation. Let $\mathbb{Z}_{+}$be the set of nonnegative integers. Let $\mathbb{Z}_{+}^{n+1}$ be the set of multi-indexes $\alpha=\left(\alpha_{0}, \alpha_{1}, \ldots, \alpha_{n}\right)$ with $\alpha_{i} \in \mathbb{Z}_{+}$, and let $|\alpha|=\sum_{i} \alpha_{i}$. For $x=\left(x_{0}, x_{1}, \ldots, x_{n}\right) \in \Delta_{n}$ and $\alpha=\left(\alpha_{0}, \alpha_{1}, \ldots, \alpha_{n}\right), \varepsilon=\left(\varepsilon_{0}, \varepsilon_{1}, \ldots, \varepsilon_{n}\right) \in$ $\mathbb{Z}_{+}^{n+1}$, let

$$
\begin{gathered}
x^{\alpha}=x_{0}^{\alpha_{0}} x_{1}^{\alpha_{1}} \cdots x_{n}^{\alpha_{n}}, \\
d x^{\varepsilon}=d x_{0}^{\varepsilon_{0}} d x_{1}^{\varepsilon_{1}} \cdots d x_{n}^{\varepsilon_{n}}=\left(d x_{0}\right)^{\varepsilon_{0}}\left(d x_{1}\right)^{\varepsilon_{1}} \cdots\left(d x_{n}\right)^{\varepsilon_{n}} .
\end{gathered}
$$

If $A=\left\{a_{1}, a_{2}, \ldots, a_{p}\right\} \subset\{0,1, \ldots, n\}$, we write

$$
\begin{gathered}
x_{A}^{\alpha}=x_{a_{1}}^{\alpha_{a_{1}}} x_{a_{2}}^{\alpha_{a_{2}}} \cdots x_{a_{p}}^{\alpha_{a_{p}}}, \\
\left(1-x_{A}\right)^{\alpha}=\left(1-x_{a_{1}}\right)^{\alpha_{a_{1}}}\left(1-x_{a_{2}}\right)^{\alpha_{a_{2}}} \cdots\left(1-x_{a_{p}}\right)^{\alpha_{a_{p}}}, \\
d x_{A}^{\varepsilon}=d x_{a_{1}}^{\varepsilon_{a_{1}}} d x_{a_{2}}^{\varepsilon_{a_{2}}} \cdots d x_{a_{p}}^{\varepsilon_{a_{p}}} .
\end{gathered}
$$

Let $\Omega_{n}(\mathbb{Z})$ be the algebra of all $\mathbb{Z}$-linear combinations of basic tame noncommutative differential forms

$$
\omega=x^{\alpha_{1}} d x^{\varepsilon_{1}} x^{\alpha_{2}} d x^{\varepsilon_{2}} \cdots x^{\alpha_{r}} d x^{\varepsilon_{r}}, \quad \alpha_{i}, \varepsilon_{i} \in \mathbb{Z}_{+}^{n+1}, i=1,2, \ldots, r,
$$

with $0 \leq x_{j} \leq 1$ and $\prod_{j=0}^{n} x_{j}=0$. These are the compatible noncommutative differential forms on the backfaces of the cube $I^{n+1}$ (see Example 2.4). If $\sum_{i}\left|\varepsilon_{i}\right|=p$ we say that $\omega$ is a $p$-form (note that 0 -forms are polynomials).

Let $\|\omega\|_{j}=\sum_{i}\left(\alpha_{i j}+\varepsilon_{i j}\right)$. The weight of $\omega$ is defined by $\|\omega\|=\max \left\{\|\omega\|_{j}: j=\right.$ $0,1, \ldots, n\}$.

Let $\Omega_{n}^{p, q}(\mathbb{Z})$ be the set of all the $p$-forms of $\Omega_{n}(\mathbb{Z})$ of weight $\|\omega\| \leq q$ and let $\Omega_{n}^{*, *}(\mathbb{Z})=\left\{\Omega_{n}^{p, q}(\mathbb{Z})\right\}_{p, q \geq 0}$.

REMARK 5.2. For all $n, p$, and $q, \Omega_{n}^{p, q}(\mathbb{Z})$ is a finitely generated free $\mathbb{Z}$-module. 
PROPOSITION 5.3. For every $n \geq 0, \Omega_{n}^{*, *}(\mathbb{Z})=\left\{\Omega_{n}^{p, q}(\mathbb{Z})\right\}_{p, q \geq 0}$ is a differential graded algebra with filtration.

Proof. We have to prove that · and $d$ on $\Omega^{*}(\mathscr{T})$ induce maps · and $d$ such that

(1) $\Omega_{n}^{p_{1}, q_{1}}(\mathbb{Z}) \otimes \Omega_{n}^{p_{2}, q_{2}}(\mathbb{Z}) \stackrel{\dot{\rightarrow}}{\rightarrow} \Omega_{n}^{p_{1}+p_{2}, q_{1}+q_{2}}(\mathbb{Z})$,

(2) $\Omega_{n}^{p, q}(\mathbb{Z}) \stackrel{d}{\rightarrow} \Omega_{n}^{p+1, q}(\mathbb{Z})$,

(3) $\Omega_{n}^{p, q}(\mathbb{Z}) \hookrightarrow \Omega_{n}^{p, q+1}(\mathbb{Z})$.

Proof OF (1). Let

$$
\begin{gathered}
\omega=\omega_{1} \cdot \omega_{2} \cdots \omega_{r} \in \Omega_{n}^{p_{1}, q_{1}}(\mathbb{Z}), \quad \omega_{i}=x^{\alpha_{i}} d x^{\varepsilon_{i}}, \quad i=1,2, \ldots, r, \\
\eta=\eta_{1} \cdot \eta_{2} \cdots \eta_{s} \in \Omega_{n}^{p_{2}, q_{2}}(\mathbb{Z}), \quad \eta_{j}=x^{\beta_{j}} d x^{\epsilon_{j}}, \quad j=1,2, \ldots, s .
\end{gathered}
$$

Then $\omega \cdot \eta=\omega_{1} \cdot \omega_{2} \cdots \omega_{r} \cdot \eta_{1} \cdot \eta_{2} \cdots \eta_{s}=\omega_{1} \cdot \omega_{2} \cdots \omega_{r} \cdot \omega_{r+1} \cdots \omega_{r+s}$ where $\omega_{r+j}=\eta_{j}\left(\left\|\omega_{r+j}\right\|_{k}=\left\|\eta_{j}\right\|_{k}\right.$ for all $j$ and $\left.k\right)$;

$$
\begin{aligned}
\|\omega \cdot \eta\|_{k} & =\sum_{l=1}^{r+s}\left\|\omega_{l}\right\|_{k}=\sum_{l=1}^{r}\left\|\omega_{l}\right\|_{k}+\sum_{l=r+1}^{r+s}\left\|\omega_{l}\right\|_{k} \\
& =\sum_{l=1}^{r}\left\|\omega_{l}\right\|_{k}+\sum_{l=1}^{s}\left\|\eta_{l}\right\|_{k} \leq\left\|\omega_{l}\right\|_{k}+\left\|\eta_{l}\right\|_{k},
\end{aligned}
$$

therefore

$$
\|\omega \cdot \eta\| \leq\left\|\omega_{l}\right\|+\left\|\eta_{l}\right\| .
$$

On the other hand, we have $\sum_{i}\left|\varepsilon_{i}\right|+\sum_{j}\left|\varepsilon_{j}\right|=p_{1}+p_{2}$. Therefore,

$$
\|\omega \cdot \eta\| \in \Omega_{n}^{p_{1}+p_{2}, q_{1}+q_{2}}(\mathbb{Z}) .
$$

Proof OF (2). Let $\omega=\omega_{1} \cdot \omega_{2} \cdots \cdots \omega_{r} \in \Omega_{n}^{p, q}(\mathbb{Z})$ with $\omega_{i}=x^{\alpha_{i}} d x^{\varepsilon_{i}} \in \Omega_{n}^{p_{i}, q_{i}}(\mathbb{Z})$, $i=1,2, \ldots, r$. Then

$$
d \omega=d\left(x^{\alpha_{i}}\right) \cdot d x^{\varepsilon_{i}}+x^{\alpha_{i}} \cdot d\left(d x^{\varepsilon_{i}}\right)=d\left(x^{\alpha_{i}}\right) \cdot d x^{\varepsilon_{i}} .
$$

Note that

$$
\begin{aligned}
d\left(x^{\alpha_{i}}\right) & =d\left(x_{0}^{\alpha_{i 0}} \cdots x_{n}^{\alpha_{i n}}\right) \\
& =\sum_{j=0}^{n} x_{0}^{\alpha_{i 0}} \cdots d\left(x_{j}^{\alpha_{i j}}\right) \cdots x_{n}^{\alpha_{i n}} \\
& =\sum_{j=0}^{n} x_{0}^{\alpha_{i 0}} \cdots x_{j-1}^{\alpha_{i, j-1}}\left(\sum_{k=1}^{n} x_{j}^{k-1} d x_{j} x_{j}^{\alpha_{i, j-k}}\right) \cdots x_{n}^{\alpha_{i n}} \\
& =\sum_{j=0}^{n} \sum_{k=1}^{n} x_{0}^{\alpha_{i 0}} \cdots x_{j-1}^{\alpha_{i, j-1}} x_{j}^{k-1} d x_{j} x_{j}^{\alpha_{i, j-k}} \cdots x_{n}^{\alpha_{i n}} .
\end{aligned}
$$

Then $\left\|d\left(x^{\alpha_{i}}\right)\right\|_{j}=\left\|x^{\alpha_{i}}\right\|_{j}$ for $j=0,1, \ldots, n$, therefore $\left\|d\left(x^{\alpha_{i}}\right)\right\|=\left\|x^{\alpha_{i}}\right\|$. Hence $\left\|d\left(x^{\alpha_{i}}\right) d x^{\varepsilon_{i}}\right\|=\left\|x^{\alpha_{i}} d x^{\varepsilon_{i}}\right\|_{j}$ and $\|d \omega\|=\|\omega\|$. On the other hand, $x^{\alpha_{i}}$ is a 0 -form, therefore $d\left(x^{\alpha_{i}}\right)$ is a 1 -form and $d \omega$ is a $(p+1)$-form, then $d \omega \in \Omega_{n}^{p+1, q}(\mathbb{Z})$. 
Proof OF (3). The proof is obvious.

Now we show that $\Omega^{*, *}(\mathbb{Z})=\left\{\Omega_{n}^{*, *}(\mathbb{Z})\right\}_{n \geq 0}$ is a simplicial algebra ( $n$ denotes the simplicial index). If $p \geq 0$ and $q \geq 1$ are fixed we consider a form $\omega \in \Omega_{n}^{p, q}(\mathbb{Z})$ as an element of $\Omega^{p}\left(\mathscr{T}_{n}\right)$ for all $n$. But $\Omega^{*}(\mathscr{T})$ is a simplicial algebra so we may restrict the face and degeneracy operators $\partial_{i}: \Omega^{p}\left(\mathscr{T}_{n}\right) \rightarrow \Omega^{p}\left(\mathscr{T}_{n+1}\right)$ and $s_{i}: \Omega^{p}\left(\mathscr{T}_{n}\right) \rightarrow \Omega^{p}\left(\mathscr{T}_{n+1}\right)$ to $\Omega_{n}^{p, q}(\mathbb{Z})$. Then we must verify that im $\partial_{i} \subset \Omega_{n-1}^{p, q}(\mathbb{Z})$ and im $s_{i} \subset \Omega_{n+1}^{p, q}(\mathbb{Z})$ for all $p \geq 0$, $q \geq 1$. Suppose that

$$
\omega=x^{\alpha_{1}} d x^{\varepsilon_{1}} x^{\alpha_{2}} d x^{\varepsilon_{2}} \cdots x^{\alpha_{r}} d x^{\varepsilon_{r}} .
$$

Then

$$
\partial_{i}(\omega)=\partial_{i}\left(x^{\alpha_{1}}\right) \partial_{i}\left(d x^{\varepsilon_{1}}\right) \partial_{i}\left(x^{\alpha_{2}}\right) \partial_{i}\left(d x^{\varepsilon_{2}}\right) \cdots \partial_{i}\left(x^{\alpha_{r}}\right) \partial_{i}\left(d x^{\varepsilon_{r}}\right),
$$

where $\partial_{i}\left(x^{\alpha_{j}}\right)=\left(\partial_{i} x_{0}\right)^{\alpha_{j 0}} \cdots\left(\partial_{i} x_{n}\right)^{\alpha_{j n}}$ and $\partial_{i}\left(d x^{\varepsilon_{j}}\right)=\left(d \partial_{i} x_{0}\right)^{\varepsilon_{j 0}} \cdots\left(d \partial_{i} x_{n}\right)^{\varepsilon_{j n}}$.

If $\varepsilon_{j i} \neq 0$, for some $j$, then $\left(d \partial_{i}\left(x_{i}\right)\right)^{\varepsilon_{j i}}=(d 1)^{\varepsilon_{j i}}=0$. Then $\partial_{i} \omega=0$ and $\left\|\partial_{i} \omega\right\| \leq\|\omega\|$. If $\varepsilon_{j i}=0$ for all $j$, it is enough to consider one block,

$$
\omega=x^{\alpha} d x^{\varepsilon}=x_{0}^{\alpha_{0}} \cdots x_{n}^{\alpha_{n}} d x_{0}^{\varepsilon_{0}} \cdots d x_{n}^{\varepsilon_{n}} \quad \text { with } \alpha_{i}, \varepsilon_{i} \in \mathbb{Z}_{+}, i=0,1, \ldots, n .
$$

Then

$$
\begin{aligned}
\partial_{i}(\omega) & =\left(\partial_{i} x_{0}\right)^{\alpha_{0}} \cdots\left(\partial_{i} x_{n}\right)^{\alpha_{n}}\left(\partial_{i} d x_{0}\right)^{\varepsilon_{0}} \cdots\left(\partial_{i} d x_{n}\right)^{\varepsilon_{n}} \\
& =x_{0}^{\alpha_{0}} \cdots x_{i-1}^{\alpha_{i-1}} 1^{\alpha_{i}} x_{i}^{\alpha_{i+1}} \cdots x_{n-1}^{\alpha_{n}} d x_{0}^{\varepsilon_{0}} \cdots d x_{i-1}^{\varepsilon_{i-1}} 1^{0} d x_{i}^{\varepsilon_{i+1}} \cdots d x_{n-1}^{\varepsilon_{n}} .
\end{aligned}
$$

Then we have

$$
\left\|\partial_{i}(\omega)\right\|_{k}= \begin{cases}\|\omega\|_{k}, & \text { for } 0 \leq k<i, \\ \|\omega\|_{k+1}, & \text { for } 0 \leq i \leq k<n, \\ 0, & \text { for } 0 \leq i<k=n .\end{cases}
$$

Therefore, $\left\|\partial_{i}(\omega)\right\| \leq\|\omega\|$. Then

$$
\partial_{i}(\omega) \in \Omega_{n-1}^{p, q}(\mathbb{Z}) .
$$

Note that if $\|\omega\|_{k}<\|\omega\|_{i}$, for all $k \neq i$, then we have a sharp inequality $\left\|\partial_{i}(\omega)\right\|<\|\omega\|$.

Similarly, for $\omega=x^{\alpha_{1}} d x^{\varepsilon_{1}} x^{\alpha_{2}} d x^{\varepsilon_{2}} \cdots x^{\alpha_{r}} d x^{\varepsilon_{r}}$, we have

$$
s_{i}(\omega)=s_{i}\left(x^{\alpha_{1}}\right) s_{i}\left(d x^{\varepsilon_{1}}\right) s_{i}\left(x^{\alpha_{2}}\right) \sigma_{i}\left(d x^{\varepsilon_{2}}\right) \cdots s_{i}\left(x^{\alpha_{r}}\right) s_{i}\left(d x^{\varepsilon_{r}}\right),
$$

where

$$
s_{i}\left(x^{\alpha_{j}}\right)=\left(s_{i} x_{0}\right)^{\alpha_{j 0}} \cdots\left(s_{i} x_{n}\right)^{\alpha_{j n}}, \quad s_{i}\left(d x^{\varepsilon_{j}}\right)=\left(d s_{i} x_{0}\right)^{\varepsilon_{j 0}} \cdots\left(d s_{i} x_{n}\right)^{\varepsilon_{j n}} .
$$

Then

$$
\begin{aligned}
s_{i}(\omega)= & \left(s_{i} x_{0}\right)^{\alpha_{0}} \cdots\left(s_{i} x_{n}\right)^{\alpha_{n}}\left(s_{i} d x_{0}\right)^{\varepsilon_{0}} \cdots\left(s_{i} d x_{n}\right)^{\varepsilon_{n}} \\
= & x_{0}^{\alpha_{0}} \cdots x_{i-1}^{\alpha_{i-1}}\left(x_{i} \cdot x_{i+1}\right)^{\alpha_{i}} x_{i+2}^{\alpha_{i+1}} \cdots x_{n+1}^{\alpha_{n+1}} \\
& \cdot d x_{0}^{\varepsilon_{0}} \cdots d\left(x_{i} \cdot x_{i+1}\right)^{\varepsilon_{i}} d x_{i+2}^{\varepsilon_{i+1}} \cdots d x_{n+1}^{\varepsilon_{n}} \\
= & x_{0}^{\alpha_{0}} \cdots x_{i-1}^{\alpha_{i-1}} x_{i}^{\alpha_{i}} x_{i+1}^{\alpha_{i}} x_{i+2}^{\alpha_{i+1}} \cdots x_{n+1}^{\alpha_{n+1}} \\
& \cdot d x_{0}^{\varepsilon_{0}} \cdots\left(d x_{i} x_{i+1}+x_{i} d x_{i+1}\right)^{\varepsilon_{i}} d x_{i+2}^{\varepsilon_{i+1}} \cdots d x_{n+1}^{\varepsilon_{n}} .
\end{aligned}
$$


Thus $s_{i}(\omega)$ is given by the expression

$$
\begin{aligned}
\sum_{j=0}^{\varepsilon_{i}}\left(\begin{array}{c}
\varepsilon_{i} \\
j
\end{array}\right) x_{0}^{\alpha_{0}} \cdots x_{i-1}^{\alpha_{i-1}} x_{i}^{\alpha_{i}} x_{i+1}^{\alpha_{i}} x_{i+2}^{\alpha_{i+1}} \cdots x_{n+1}^{\alpha_{n+1}} \\
\quad \cdot d x_{0}^{\varepsilon_{0}} \cdots d x_{i}^{\varepsilon_{i-j}} x_{i+1}^{\varepsilon_{i-j}} x_{i}^{j} d x_{i+1}^{j} d x_{i+2}^{\varepsilon_{i+1}} \cdots d x_{n+1}^{\varepsilon_{n}} .
\end{aligned}
$$

Note that $\left\|s_{i}(\omega)\right\|_{k}=\alpha_{i}+\varepsilon_{i}-j+j=\|\omega\|_{i}$ for $k=i, i+1$, and $\left\|s_{i}(\omega)\right\|_{k}=\alpha_{k-1}+$ $\varepsilon_{k-1}=\|\omega\|_{k-1}$ for $k>i+1$. Then

$$
\left\|s_{i}(\omega)\right\|_{k}= \begin{cases}\|\omega\|_{k}, & \text { for } k<i \\ \|\omega\|_{i}, & \text { for } k=i, i+1 \\ \|\omega\|_{k-1}, & \text { for } k>i+1\end{cases}
$$

Therefore, $\left\|s_{i}(\omega)\right\| \leq\|\omega\|$ and $s_{i}(\omega) \in \Omega_{n+1}^{p, q}(\mathbb{Z})$.

From the definition and Proposition 4.7 it follows that $\partial_{i}$ and $s_{i}$ are morphisms of DGA's. Then we have the following proposition.

Proposition 5.4. The algebra $\Omega^{*, *}(\mathbb{Z})=\left\{\Omega_{n}^{*, *}(\mathbb{Z})\right\}_{n \geq 0}$ is a simplicial DGA.

Now consider $0 \leq x_{j} \leq 1$ for $j=0,1, \ldots, n$ with $\prod_{j} x_{j}=0$. Define

$$
\Omega^{p, q}\left(\Delta_{n}\right)=\Omega_{n}^{p, q}(\mathbb{Z}) \otimes_{\mathbb{Z}} \mathbb{Q}_{q},
$$

where $\mathbb{Q}_{q}=\mathbb{Z}[1 / 2, \ldots, 1 / q]$, for $q>1$, and $\mathbb{Q}_{0}=\mathbb{Q}_{1}=\mathbb{Z}$.

Let $\Delta_{n}^{k}, 0 \leq k \leq n$, be the $k$-skeleton of $\Delta_{n}$, and let

$$
\Omega^{p, q}\left(\Delta_{n}, \Delta_{n}^{k}\right)=\left\{\omega \in \Omega^{p, q}\left(\Delta_{n}\right):\left.\omega\right|_{\Delta_{n}^{k}} \equiv 0\right\}
$$

Let $\Omega^{p, q}\left(\Delta_{n}^{k}\right)$ be the set of all $\mathbb{Q}_{q}$-linear combinations of forms which are nonzero on exactly one $k$-face of $\Delta_{n}^{k}$.

Proposition 5.5. The sequence

$$
\Omega^{p, q}\left(\Delta_{n}, \Delta_{n}^{k-1}\right) \stackrel{r \text { restrictions }}{\longrightarrow} \Omega^{p, q}\left(\Delta_{n}^{k}, \Delta_{n}^{k-1}\right) \longrightarrow 0
$$

is an exact sequence of $\mathbb{Q}_{q}$-modules for all $p \geq 0, q \geq 1$.

Proof. Let $\omega \in \Omega^{p, q}\left(\Delta_{n}, \Delta_{n}^{k-1}\right)$ with $q \geq 1$. Then the form $\omega$ is a linear combination $\omega=\sum_{i} \omega_{i}$, where each $\omega_{i}$ is nonzero on exactly one face of $\Delta_{n}^{k}$. Let $F$ be such a face. Then

$$
\left.\omega\right|_{G} \equiv 0 \quad \text { if } G \text { is any } k \text {-face of } \Delta_{n}^{k} \text { different from } F \text {. }
$$

We write $F=F(A, B)$ (using the notation of Example 2.4) where $A$ and $B$ are two disjoint sets $A=\left\{a_{1}, a_{2}, \ldots, a_{k+1}\right\}$ and $B=\left\{b_{1}, b_{2}, \ldots, b_{n-k}\right\}$ such that $0 \leq a_{1}<a_{2}<\cdots<$ 
$a_{k+1} \leq n, 0 \leq b_{1}<b_{2}<\cdots<b_{n-k} \leq n, A \cup B=\{0,1, \ldots, n\}, 0 \leq x_{i} \leq 1$, for all $i \in A$, $\prod_{i \in A} x_{i}=0$, and $x_{j} \equiv 1$ for all $j \in B$.

We have $\left.\omega\right|_{\Delta_{n}^{k-1}} \equiv 0$. Therefore $\omega$ is a linear combination of forms of the type

$$
f^{1}(x) d x^{\varepsilon_{1}} \cdots f^{s}(x) d x^{\varepsilon_{s}},
$$

where $f^{j}(x)=x_{A}^{\alpha_{1}}\left(1-x_{A}\right)^{\alpha_{2}} x_{B}^{\beta_{1}}\left(1-x_{B}\right)^{\beta_{2}} f_{j}(x)\left(f_{j}(x)\right.$ is a polynomial in $\left.x\right)$. For $F$ we may assume that $\left|\beta_{2}\right|=0$. Thus $f^{j}(x)=x_{A}^{\alpha_{1}}\left(1-x_{A}\right)^{\alpha_{2}} x_{B}^{\beta_{1}} f_{j}(x)$. Note that if $\left|\alpha_{i}\right|=0$ for $i=1,2$ (and for all $j$ ), then $\varepsilon_{i t} \neq 0$ for some $t$.

On $F$ we have $f^{j}(x)=x_{A}^{\alpha_{1}}\left(1-x_{A}\right)^{\alpha_{2}} f_{j}\left(x_{A}\right)$.

Let $G=G\left(A^{\prime}, B^{\prime}\right)$ be another $k$-face of $\Delta_{n}^{k}$. If $A=A^{\prime}$, then there exists $i \in B$ with $x_{i} \equiv 0$, then $f^{j}(x)=0$ and $\left.\omega\right|_{G} \equiv 0$.

If $A \neq A^{\prime}$ then there exists $i \in A$ such that $x_{i} \equiv 1$. Then either $f^{j}(x) \equiv 0$ or $d x_{i}^{\varepsilon_{i t}}=0$ for some $t$, in both cases we have $\left.\omega\right|_{G} \equiv 0$.

Let $\phi(x)=x_{B}$, then $\phi \in \Omega^{0,1}\left(\Delta_{n}\right)$. Define $\omega_{k}=\phi \cdot \omega$. Note that there exists $i \in A$ such that $\left\|\omega_{k}\right\|_{j}=1 \leq\left\|\omega_{k}\right\|_{i}=q$ for all $j \in B$. Therefore $\omega_{k} \in \Omega^{p, q}\left(\Delta_{n}\right)$. Moreover,

$$
\left.\omega_{k}\right|_{F(A, B)}=\left.\left.\phi\right|_{F(A, B)} \cdot \omega\right|_{F(A, B)} \equiv 1 \cdot \omega=\omega
$$

If $F\left(A^{\prime}, B^{\prime}\right)$ is another $k$-face of $\Delta_{n}^{k}$, then we have

$$
\left.\omega_{k}\right|_{F\left(A^{\prime}, B^{\prime}\right)}=\left.\left.\left.\phi\right|_{F\left(A^{\prime}, B^{\prime}\right)} \cdot \omega\right|_{F\left(A^{\prime}, B^{\prime}\right)} \equiv \phi\right|_{F\left(A^{\prime}, B^{\prime}\right)} \cdot 0=0 .
$$

If $F(E, H)$ is a $(k-1)$-face of $\Delta_{n}^{k}$, then there exists at least one $i \in A$ such that $i \notin E$. Then $x_{i}$ is either 0 or 1 on $F(E, H)$. Therefore $\left.\omega\right|_{F(E, H)}=0$ and then $\left.\omega_{k}\right|_{F(E, H)} \equiv 0$. We deduce that $\omega_{k} \in \Omega^{p, q}\left(\Delta_{n}, \Delta_{n}^{k-1}\right)$ and $\left.\omega_{k}\right|_{\Delta_{n}^{k}} \equiv \omega$.

Proposition 5.6. The sequence

$$
\Omega^{p, q}\left(\Delta_{n}\right) \stackrel{r \text { restrictions }}{\longrightarrow} \Omega^{p, q}\left(\partial \Delta_{n}\right) \longrightarrow 0
$$

is an exact sequence of $\mathbb{Q}_{q}$-modules for all $p \geq 0, q \geq 1$.

Proof. For $k=0$, the sequence

$$
\Omega^{p, q}\left(\Delta_{n}\right) \longrightarrow \Omega^{p, q}\left(\Delta_{n}^{0}\right) \longrightarrow 0
$$

is exact, thus any element $a \in \mathbb{Q}_{q}$ can be pulled back to the form $\omega\left(x_{0}, \ldots, x_{n}\right)=a$, that is, it is a constant $\mathbb{Q}_{q}$-polynomial.

Assume by induction that

$$
\Omega^{p, q}\left(\Delta_{n}\right) \longrightarrow \Omega^{p, q}\left(\partial \Delta_{n}^{k-1}\right) \longrightarrow 0
$$


is exact. Consider the following commutative diagram:

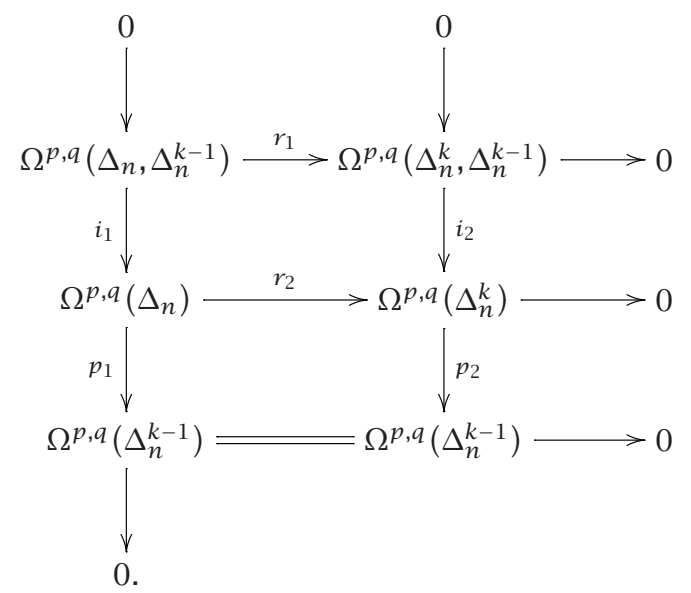

The left column is exact by induction hypothesis (and by the definition of $\Omega^{p, q}\left(\Delta_{n}^{k}\right.$, $\left.\Delta_{n}^{k-1}\right)$ ). The right column is exact by definition. The first row is exact by Proposition 5.5 . We show that the second row is exact. Let $\omega \in \Omega^{p, q}\left(\Delta_{n}^{k}\right)$.

CASE 1. If $\omega \in \operatorname{ker} p_{2}$, then there exists $\omega^{\prime} \in \Omega^{p, q}\left(\Delta_{n}^{k}, \Delta_{n}^{k-1}\right)$ with $i_{2}\left(\omega^{\prime}\right)=\omega$. But the first row is exact, then there exists $\omega^{\prime \prime} \in \Omega^{p, q}\left(\Delta_{n}, \Delta_{n}^{k-1}\right)$ such that $\omega^{\prime \prime}=r_{1}\left(\omega^{\prime}\right)$. Hence $\omega=i_{2}\left(r_{1}\left(\omega^{\prime}\right)\right)=r_{2}\left(i_{1}\left(\omega^{\prime}\right)\right)$ and $\omega \in \operatorname{im} r_{2}$.

CASE 2. If $p_{2}(\omega) \neq 0$, then there exists $\omega^{\prime} \in \Omega^{p, q}\left(\Delta_{n}\right)$ such that $p_{1}\left(\omega^{\prime}\right)=p_{2}(\omega)$. Then

$$
\begin{aligned}
p_{2}\left(\omega-r_{2}\left(\omega^{\prime}\right)\right) & =p_{1}(\omega) p_{2}\left(r_{2}\left(\omega^{\prime}\right)\right) \\
& =p_{1}\left(\omega^{\prime}\right)-p_{2}\left(r_{2}\left(\omega^{\prime}\right)\right) \\
& =0,
\end{aligned}
$$

thus $\omega^{\prime}-r_{2}\left(\omega^{\prime}\right) \operatorname{ker} p_{2}$, then $\omega^{\prime}-r_{2}\left(\omega^{\prime}\right) \in \operatorname{im} r_{2}$ (by Case 1), then $\omega \in \operatorname{im} r_{2}$.

Finally for $k=n-1$, we have $\Delta_{n}^{n-1}=\partial \Delta_{n}$. Thus the sequence

$$
\Omega^{p, q}\left(\Delta_{n}\right) \longrightarrow \Omega^{p, q}\left(\partial \Delta_{n}\right) \longrightarrow 0
$$

is exact.

Now we prove that, for any $q \geq 0$ the complex $\Omega^{*, q}\left(\Delta_{n}\right)$ has trivial cohomology. If $\lambda: \mathscr{T} \rightarrow \mathbb{Z}$ is any linear form with $\lambda(1)=1$. For each $p \geq 0$, let $J_{\lambda}: \Omega^{p}(\mathscr{T}) \rightarrow \Omega^{p-1}(\mathscr{T})$ be the map defined at $\omega=f^{0} d f^{1} \cdots d f^{p} \in \Omega^{p}(\mathscr{T})$ by

$$
J_{\lambda}(\omega)=\lambda\left(f^{0}\right) f^{1} d f^{2} \cdots d f^{P}-\lambda\left(f^{0} f^{1}\right) d f^{2} \cdots d f^{p} .
$$

Then $J_{\lambda}$ is a contracting homotopy (Lemma 4.5). Consider $\Omega^{p, q}\left(\Delta_{n}\right)$ as a submodule of $\Omega^{p}\left(\mathscr{T}_{n}\right)$ and $j_{\lambda}$ restricted to $\Omega^{p, q}\left(\Delta_{n}\right)$. Suppose that

$$
\omega=x^{\alpha_{1}} d x^{\varepsilon_{1}} \cdots x^{\alpha_{r}} d x^{\varepsilon_{r}}=\sum f^{0} d f^{1} \cdots d f^{p} .
$$


Note that $\left\|j_{\lambda}(\omega)\right\|_{j} \leq\|\omega\|_{j}$ for all $j$. Therefore $\left\|j_{\lambda}(\omega)\right\| \leq\|\omega\| \leq q$ and $j_{\lambda}(\omega) \in$ $\Omega^{p-1, q}\left(\Delta_{n}\right)$. Thus $j_{\lambda}: \Omega^{p, q}\left(\Delta_{n}\right) \rightarrow \Omega^{p-1, q}\left(\Delta_{n}\right)$ is a contracting homotopy (by Lemma 4.5).

If $\omega \in \Omega^{p, q}\left(\Delta_{n}\right)$ and $d \omega=0$ we say that $\omega$ is closed. We say that $\omega$ is exact if there exists $\eta \in \Omega^{p-1, q}\left(\Delta_{n}\right)$ such that $\omega=d \eta$. Then we have the following lemma.

LEMMA 5.7 (the noncommutative tame Poihcaré lemma). If $\omega \in \Omega^{p, q}\left(\Delta_{n}\right)$ is a closed form, then $\omega$ is exact.

6. Integration of noncommutative tame forms. In this section, we introduce integration of noncommutative tame forms, prove the tame noncommutative Stokes' theorem, and use this result to define a morphism of $\mathbb{Q}_{q}$-modules $I: \Omega^{*, q}\left(\Delta_{n}\right) \rightarrow$ $C^{*}\left(\Delta_{n} ; \mathbb{Q}_{q}\right)$ which plays an important role in the proof of the de Rham theorem. The definition of the integral of noncommutative tame forms is motivated by the ideas presented in [12].

Let $\Omega^{*, *}\left(\Delta_{n}\right)$ denote the algebra of noncommutative tame differential forms in the variables $x_{0}, x_{1}, \ldots, x_{n}$.

Let $T^{*, *}\left(\Delta_{n}\right)$ be the algebra of differential forms of Cenkl-Porter with $\mathbb{Q}_{q}$-coefficients on the standard cube $I^{n+1} \subset \mathbb{R}^{n+1}$. Define $F: \Omega^{p, q}\left(\Delta_{n}\right) \rightarrow T^{p, q}\left(\Delta_{n}\right)$ as follows: if $\omega \in$ $\Omega^{0, q}\left(\Delta_{n}\right)$ or $\omega=d x_{j} \in \Omega^{1,1}\left(\Delta_{n}\right)$ then $F(\omega)=\omega$; if $\omega=f^{0} d f^{1} \cdots d f^{p} \in \Omega^{p, q}\left(\Delta_{n}\right)$ for $p>1$, then $F\left(f^{0} d f^{1} \cdots d f^{p}\right)=f^{0} d f^{1} \wedge \cdots \wedge d f^{p}$. Then $F: \Omega^{p, q}\left(\Delta_{n}\right) \rightarrow T^{p, q}\left(\Delta_{n}\right)$ defines a morphism of $\mathbb{Q}_{q}$-modules.

Note that for all $p \geq 0$, we have

$$
F\left(f^{0} d f^{1} \cdots d f^{p}\right)=F\left(f^{0}\right) F\left(d f^{1}\right) \wedge \cdots \wedge F\left(d f^{p}\right) .
$$

In particular, if $\omega \in \Omega^{p_{1}, q_{1}}\left(\Delta_{n}\right)$ and $\eta \in \Omega^{p_{2}, q_{2}}\left(\Delta_{n}\right)$ then

$$
F(\omega \cdot \eta)=F(\omega) \wedge F(\eta)
$$

In other words $F$ is a morphism of algebras. To prove this identity it is enough to consider $\omega=f^{0} d f^{1}, \eta=g^{0} d g^{1} \in \Omega^{1}\left(\mathcal{T}_{n}\right)$. Then

$$
\begin{aligned}
F(\omega \cdot \eta) & =F\left(f^{0} d f^{1} \cdot g^{0} d g^{1}\right) \\
& =F\left(f^{0} d\left(f^{1} g^{0}\right) d g^{1}-f^{0} f^{1} d g^{0} d g^{1}\right) \\
& =f^{0} d\left(f^{1} g^{0}\right) \wedge d g^{1}-f^{0} f^{1} d g^{0} d g^{1} \\
& =f^{0} d f^{1} g^{0} \wedge d g^{1}+f^{0} f^{1} d g^{0} \wedge d g^{1}-f^{0} f^{1} d g^{0} \wedge d g^{1} \\
& =f^{0} g^{0} d f^{1} \wedge d g^{1} \\
& =F(\omega) \wedge F(\eta) .
\end{aligned}
$$

Similarly, the following propositions can be proved by direct computations. 
Proposition 6.1. The diagram

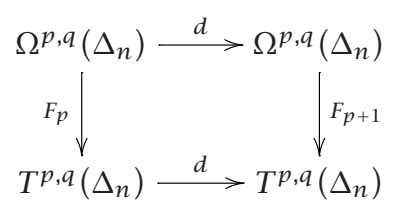

commutes for all $p \geq 0$.

Proposition 6.2. Let $\omega=f^{0} d f^{1} \cdots d f^{p} \in \Omega^{p, q}\left(\Delta_{n}\right)$. If $p>n$ then $F(\omega)=0$. If $0<p \leq n$ and $G_{p}$ denotes the permutation group of the set $\{1,2, \ldots, p\}$, then

$$
F(\omega)=\sum_{0 \leq j_{1}<\cdots<j_{p} \leq n} \sum_{\tau \in \mathscr{G}_{p}} \operatorname{sgn} \tau f^{0} \frac{\partial f^{1}}{\partial x_{j_{\tau}(1)}} \cdots \frac{\partial f^{p}}{\partial x_{j_{\tau(p)}}} d x_{j_{1}} \wedge \cdots \wedge d x_{j_{p}} .
$$

Proposition 6.3. Suppose that $\omega=x^{\alpha_{1}} d x^{\varepsilon_{1}} \cdots x^{\alpha_{r}} d x^{\varepsilon_{r}} \in \Omega_{n}^{p, q}\left(\Delta_{n}\right)$ and $0<p \leq$ $n$, then

(1) if $\sum_{i} \varepsilon_{i j} \geq 2$ for some $j$, then $F(\omega)=0$;

(2) if $0 \leq i_{1}<\cdots<i_{p} \leq n$ and $\tau \in \mathscr{G}_{p}$ such that $\tau(1)<\cdots<\tau(p)$ and $\sum_{i} \varepsilon_{i \tau(j)} \leq 1$ for all $j$, then

$$
F(\omega)=\operatorname{sgn} \tau x^{\alpha_{1}} \cdots x^{\alpha_{r}} d x_{i_{\tau(1)}} \wedge \cdots \wedge d x_{i_{\tau(p)}} .
$$

REMARK 6.4. Proposition 6.5 implies that $F$ is a simplicial map.

From Proposition 3.1, we obtain the following result.

Proposition 6.5. Let $p \geq 0, q \geq 1$. If $\omega \in \Omega_{n}^{p, q}(\mathbb{Z})$ and if $G$ is a $p$-face of $\Delta_{n}$, then

$$
\int_{G} F(\omega) \in \mathbb{Q}_{q}
$$

If $\omega=\Omega^{p, q}\left(\Delta_{n}\right)$ and $\sigma: \Delta_{p} \rightarrow \Delta_{n}$ is a $p$-simplex, we define the integral of $\omega$ on $\sigma$ by

$$
\int_{\sigma} \omega=\int_{\sigma} F(\omega)=\int_{\Delta_{p}} \sigma^{*} F(\omega)
$$

If $\sigma=\sum_{i} \sigma_{i} \otimes a_{i} \in C_{p}\left(\Delta_{n} ; \mathbb{Q}_{q}\right)$, then the integral of $\omega$ on $\sigma$ is defined by

$$
\int_{\sigma} \omega=\sum_{i} a_{i} \int_{\sigma_{i}} \omega
$$

Proposition 6.6 (noncommutative Stokes' theorem). Let $\sigma$ be a p-chain on $\Delta_{n}$ and let $\omega \in \Omega^{p, q}\left(\Delta_{n}\right)$. Then

$$
\int_{\sigma} d \omega=\int_{\partial \sigma} \omega
$$

Proof. Let $\omega \in \Omega^{p, q}\left(\Delta_{n}\right)$ and let $\sigma: \Delta_{p} \rightarrow \Delta_{n}$ be a $p$-simplex. By Proposition 6.1 and by the classical Stokes' theorem, we get

$$
\int_{\sigma} d \omega=\int_{\sigma} F(d \omega)=\int_{\sigma} d(F(\omega))=\int_{\partial \sigma} F(\omega)=\int_{\partial \sigma} \omega .
$$


Let $\left(C^{*}\left(\Delta_{n} ; \mathbb{Q}_{q}\right), \delta\right)$ denote the standard complex of cochains on $\Delta_{n}$ with coefficients in $\mathbb{Q}_{q}$. Let

$$
I: \Omega^{*, q}\left(\Delta_{n}\right) \longrightarrow C^{*}\left(\Delta_{n} ; \mathbb{Q}_{q}\right)
$$

be the morphism of $\mathbb{Q}_{q}$-modules defined as follows: given $\sigma \in C_{p}\left(\Delta_{n} ; \mathbb{Q}_{q}\right)$ and $\omega \in$ $\Omega^{p, q}\left(\Delta_{n}\right)$,

$$
I_{p}(\omega)(\sigma)=\int_{\sigma} \omega
$$

The Stokes theorem implies that $I$ is a map of cochain complexes. We also have that the diagram

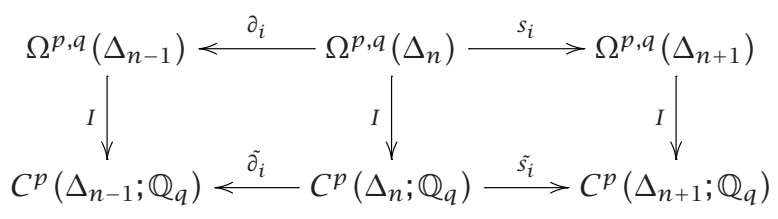

commutes for $0 \leq i \leq n$. Then $I$ is a simplicial map.

Proposition 6.7. The diagram

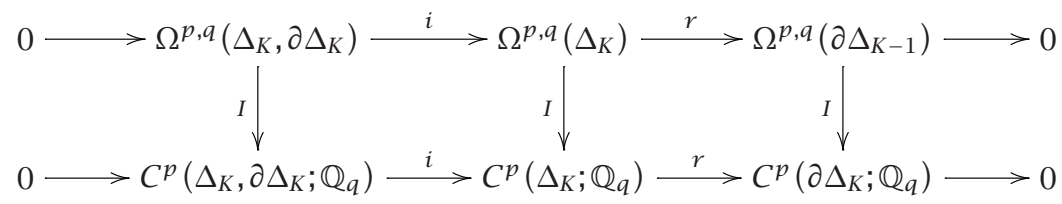

commutes for all $p \geq 0, q \geq 1$. (The i's and $r$ 's denote the inclusions and restrictions, respectively.)

Proof. Let $\sigma \in C_{p}\left(\Delta_{k} ; \mathbb{Q}_{q}\right)$ and $\omega \in \Omega^{p, q}\left(\Delta_{k}, \partial \Delta_{k}\right)$, then $r(\omega)=r(i(\omega))$. Therefore,

$$
\begin{aligned}
I(i(\omega))(\sigma) & =\int_{\sigma} i(\omega)=\int_{\sigma \cap \partial \Delta_{k}} \omega+\int_{\sigma-\left(\sigma \cap \sigma \Delta_{k}\right)} \omega \\
& =\int_{\partial-\left(\sigma \cap \partial \Delta_{k}\right)} \omega=i(I(\omega))(\sigma) .
\end{aligned}
$$

On the other hand, we have

$$
\begin{aligned}
I(r(\omega))(\sigma) & =I(\sigma)(r(\omega))=\int_{\sigma} r(\omega)=\left.\int_{\sigma} \omega\right|_{\partial \Delta_{k}} \\
& =\int_{\sigma \cap \partial \Delta_{k}} \omega=\int_{r(\sigma)} \omega=I(r(\sigma))(\omega) .
\end{aligned}
$$

7. The noncommutative tame de Rham theorem for cohomology. In this section, we introduce the noncommutative de Rham complex of Cenkl and Porter for a simplicial set of finite type $X$. Then we use the noncommutative versions of the Poincaré lemma and the Stokes' theorem to prove the noncommutative tame de Rham theorem. 
Let $X$ be a simplicial complex of finite type. Let $X_{n}$ be the collection of nondegenerated $n$-simplices in $X$. A noncommutative differential form of type $(p, q)$ on $X$ is a simplicial map $\omega: X_{n} \rightarrow \Omega^{p, q}\left(\Delta_{n}\right)$ (in other words, $\omega$ is a map such that for $G \in X_{n}$ and any face $F$ of $G, \omega(F)$ is the restriction of $\omega(G)$ to $F$ ). The collection of all such forms is denoted by $\Omega^{p, q}(X)$.

For a $p$-chain $\sigma=\sum_{i} \sigma_{i} \otimes a_{i} \in C_{p}\left(X ; \mathbb{Q}_{q}\right), \sigma_{i}: \Delta_{p} \rightarrow X$ and $\omega \in \Omega^{p, q}(X)$, we define

$$
\int_{\sigma} \omega=\left.\sum_{i} a_{i} \int_{\Delta_{p}} \omega\right|_{\sigma_{i}}
$$

so we may define the map $I: \Omega^{p, q}(X) \rightarrow C^{p}\left(X ; \mathbb{Q}_{q}\right)$ by

$$
I_{p}(\omega)(\sigma)=\int_{\sigma} \omega
$$

Then

$$
\delta I_{p}(\omega)(\sigma)=I_{p}(\omega)(\partial \sigma)=\left.\int_{\partial \Delta_{p}} \omega\right|_{\partial \sigma}
$$

On the other hand,

$$
I_{p+1}(d \omega)(\sigma)=I_{p}(d \omega)(\sigma)=\left.\int_{\Delta_{p}} d \omega\right|_{\sigma}
$$

Thus integration induces a map of cochain complexes. Then we have the following theorem.

THEOREM 7.1. Let $X$ be a simplicial set of finite type. Then for $q \geq 1$ the map

$$
I: H^{i}\left(\Omega^{*, q}(X)\right) \stackrel{\cong}{\longrightarrow} H^{i}\left(X ; \mathbb{Q}_{q}\right),
$$

induced by integration, is an isomorphism of $\mathbb{Q}_{q}$-modules for all $i \geq 0$.

Proof. Induction on the skeleta of $X$. For $k=0$ the statement is true because

$$
H^{i}\left(\Omega^{*, q}(X)\right)=\left\{\begin{array}{ll}
\mathbb{Q}_{q}, & \text { if } i=0, \\
0, & \text { if } i>0,
\end{array} \quad H^{i}\left(X ; \mathbb{Q}_{q}\right)= \begin{cases}\mathbb{Q}_{q}, & \text { if } i=0, \\
0, & \text { if } i>0\end{cases}\right.
$$

Suppose that the statement is true on the $\ell$-skeleton, $X_{\ell}$ of $X$ for $\ell<k$.

From Proposition 6.7 it follows that the diagram

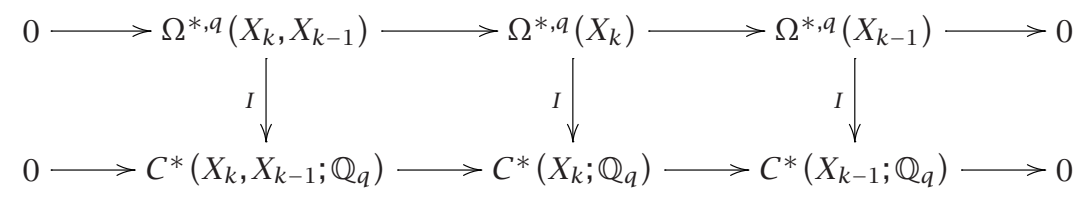

is commutative. Then the following diagram commutes: 


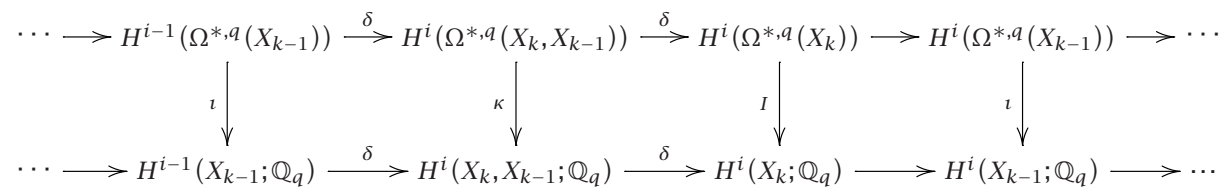

The rows are exact, an $\imath$ is an isomorphism by assumption. We prove that $\kappa$ is an isomorphism. Let $\left\{\Delta_{k, j}: j \in J\right\}$ be the set of $k$-simplices of $X_{k}$. Then

$$
\begin{aligned}
\Omega^{*, q}\left(X_{k}, X_{k-1}\right) & \cong \bigoplus_{j} \Omega^{*, q}\left(\Delta_{k, j}, \partial \Delta_{k, j}\right), \\
C^{*}\left(X_{k}, X_{k-1} ; \mathbb{Q}_{q}\right) & \cong \bigoplus_{j} C^{*}\left(\Delta_{k, j}, \partial \Delta_{k, j} ; \mathbb{Q}_{q}\right)
\end{aligned}
$$

are isomorphisms of $\mathbb{Q}_{q}$-modules. Then it is enough to prove that integration induces an isomorphism

$$
I: H^{i}\left(\Omega^{*, q}\left(\Delta_{k}, \partial \Delta_{k}\right)\right) \rightarrow H^{i}\left(C^{*}\left(\Delta_{k}, \partial \Delta_{k} ; \mathbb{Q}_{q}\right)\right)
$$

Consider the following commutative diagram (Proposition 6.7):

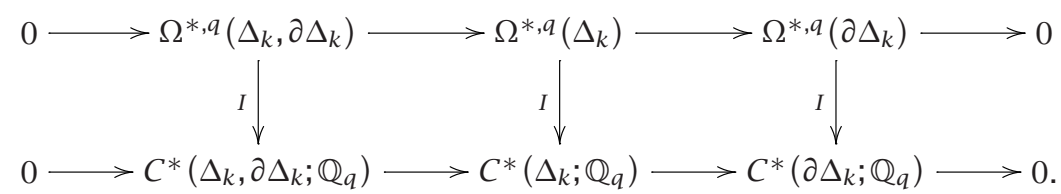

The first row is exact by Proposition 5.6.

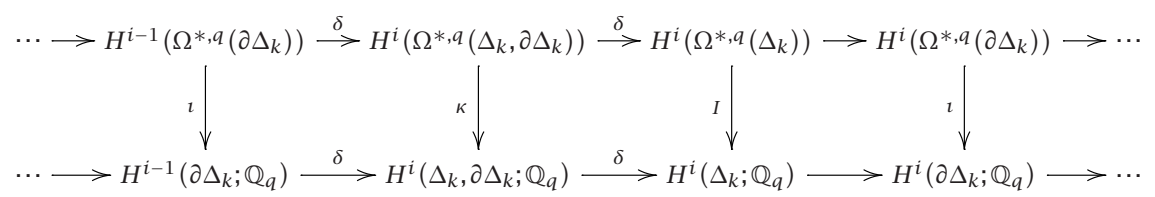

Therefore $I$ is an isomorphism by lemma five ("so named because of the five-term exact sequence involved in its formulation,” Spanier [21, page 185]).

In the following examples, we consider $X$ to be the circle $S^{1}$ and we verify the isomorphism $H^{*}\left(\Omega^{*, q}(X)\right) \cong H^{*}\left(X ; \mathbb{Q}_{q}\right)$ (for $\left.q=1,2\right)$ by computing directly $H^{i}\left(\Omega^{*, q}(X)\right)$.

EXAMPLE 7.2. In this example, we consider the complex of noncommutative tame differential forms of weight $\leq 1$ on the circle $\Omega^{*, 2}\left(S^{1}\right)$, where $\Omega^{*, 2}\left(S^{1}\right)$ denotes the complex of noncommutative differential forms of weight $\leq 2$ on the circle. In this case the ground ring is $\mathbb{Q}_{2}=\mathbb{Z}[1 / 2]$. 


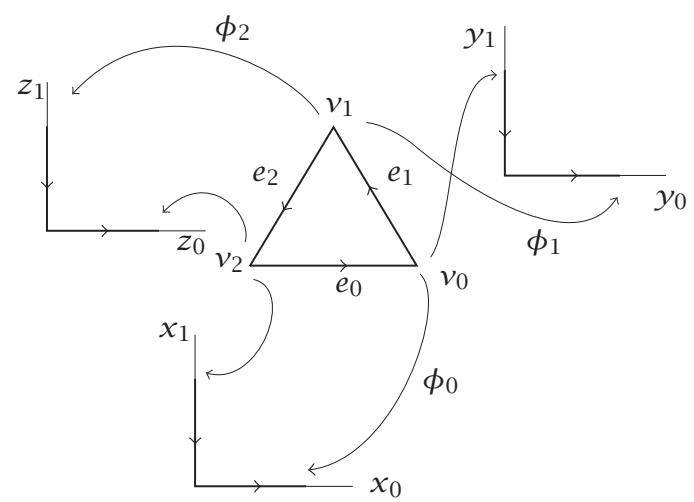

FIGURE 7.1. Differential forms on $S^{1}$.

Consider the triangulation of $S^{1}$ with 0 -simplexes $v_{0}, v_{1}, v_{2}$, and 1-simplexes $e_{0}, e_{1}$, $e_{2}$ oriented as indicated in Figure 7.1. We can consider each 1-simplex $e_{i}$ is the image of a continuous injective function $\psi_{i}: \Delta_{1} \rightarrow S^{1}$. Let $\phi_{i}=\psi^{-1}: e_{i} \rightarrow \Delta_{1}, i=0,1,2$. Recall that the 1-simplex $\Delta_{1}$ is considered to be the union of the two backfaces of the 2-cube $I^{2}$ in $\mathbb{R}^{2}$, as in Example 2.4.

A form $\eta \in \Omega^{0,2}\left(S^{1}\right)$ is a map such that $\eta\left(e_{i}\right)=\eta_{i} \in \Omega^{0,2}\left(\Delta_{1}\right)=\Omega_{1}^{0,2}(\mathbb{Z}) \otimes \mathbb{Q}_{2}$, satisfying

$$
\begin{aligned}
& \eta_{0}\left(\phi_{0}\left(v_{0}\right)\right)=\eta_{1}\left(\phi_{1}\left(v_{0}\right)\right), \\
& \eta_{1}\left(\phi_{1}\left(v_{1}\right)\right)=\eta_{2}\left(\phi_{2}\left(v_{1}\right)\right), \\
& \eta_{2}\left(\phi_{2}\left(v_{2}\right)\right)=\eta_{0}\left(\phi_{0}\left(v_{2}\right)\right) .
\end{aligned}
$$

Note that $x_{0} x_{1}=y_{0} y_{1}=z_{0} z_{1}=0$, therefore we have

$$
\begin{gathered}
\eta_{0}\left(x_{0}, x_{1}\right)=k_{0}+a_{0} x_{0}+a_{1} x_{1}+a_{2} x_{0}^{2}+a_{3} x_{1}^{2}, \\
\eta_{1}\left(y_{0}, y_{1}\right)=k_{1}+b_{0} y_{0}+b_{1} y_{1}+b_{2} y_{0}^{2}+b_{3} y_{1}^{2}, \\
\eta_{2}\left(z_{0}, z_{1}\right)=k_{2}+c_{0} z_{0}+c_{1} z_{1}+c_{2} z_{0}^{2}+c_{3} z_{1}^{2},
\end{gathered}
$$

with $k_{i}, a_{j}, b_{j}, c_{j} \in \mathbb{Q}_{2}$ for $i=0,1,2$ and $j=0,1,2,3$, such that

$$
\begin{aligned}
& k_{0}+a_{0}+a_{2}=k_{1}+b_{1}+b_{3}, \\
& k_{1}+b_{0}+b_{2}=k_{2}+c_{1}+c_{3}, \\
& k_{2}+c_{0}+c_{2}=k_{0}+a_{1}+a_{3} .
\end{aligned}
$$

If $\eta$ is closed then $d \eta_{i}=0$ for $i=0,1,2$, then $a_{j}=b_{j}=c_{j}=0$. Then (7.15) implies $k_{0}=k_{1}=k_{2}=0$ and $\eta$ is constant. Then $H^{0}\left(\Omega^{*, 2}\left(S^{1}\right)\right) \cong \mathbb{Q}_{2}$.

Now let $\omega \in \Omega^{1,2}\left(S^{1}\right)$. Then $\omega\left(e_{i}\right)=\omega_{i} \in \Omega^{1,2}\left(\Delta_{1}\right)$. We have

$$
\begin{aligned}
& \omega_{0}\left(x_{0}, x_{1}\right)=a_{0} d x_{0}+a_{1} d x_{1}+a_{2} x_{0} d x_{0}+a_{3} d x_{0} x_{0}+a_{4} x_{1} d x_{1}+a_{5} d x_{1} x_{1}, \\
& \omega_{1}\left(y_{0}, y_{1}\right)=b_{0} d y_{0}+b_{1} d y_{1}+b_{2} y_{0} d y_{0}+b_{3} d y_{0} y_{0}+b_{4} y_{1} d y_{1}+b_{5} d y_{1} y_{1}, \\
& \omega_{2}\left(z_{0}, z_{1}\right)=c_{0} d z_{0}+c_{1} d z_{1}+c_{2} z_{0} d z_{0}+c_{3} d z_{0} z_{0}+c_{4} z_{1} d z_{1}+c_{5} d z_{1} z_{1} .
\end{aligned}
$$


Then

$$
\begin{aligned}
& d \omega_{0}=a_{2} d x_{0}^{2}-a_{3} d x_{0}^{2}+a_{4} d x_{1}^{2}-a_{5} d x_{1}^{2}, \\
& d \omega_{1}=b_{2} d y_{0}^{2}-b_{3} d y_{0}^{2}+b_{4} d y_{1}^{2}-b_{5} d y_{1}^{2}, \\
& d \omega_{2}=c_{2} d z_{0}^{2}-c_{3} d z_{0}^{2}+c_{4} d z_{1}^{2}-c_{5} d z_{1}^{2} .
\end{aligned}
$$

If $\omega$ is closed we have $a_{i}=a_{i+1}, b_{i}=b_{i+1}$, and $c_{i}=c_{i+1}$, for $i=2,4$. Then we have $\operatorname{dim} \operatorname{ker}\left(d: \Omega^{1,2}\left(S^{1}\right) \rightarrow \Omega^{2,2}\left(S^{1}\right)\right)=12$.

Note that the linear system (7.15) is equivalent to

$$
\begin{gathered}
k_{0}-k_{1}=b_{1}+b_{3}-a_{0}-a_{2}, \\
k_{1}-k_{2}=c_{1}+c_{3}-b_{0} b_{2}, \\
0=a_{0}-a_{1}+a_{2}-a_{3}+b_{0}-b_{1}+b_{2}-b_{3}+c_{0}-c_{1}+c_{2}-c_{3} .
\end{gathered}
$$

Then $\operatorname{dimim}\left(d: \Omega^{0,2}\left(S^{1}\right) \rightarrow \Omega^{1,2}\left(S^{1}\right)\right)=11$ and $H^{1}\left(\Omega^{*, 2}\left(S^{1}\right)\right) \cong \mathbb{Q}_{2}$.

Now let $\theta \in \Omega^{2,2}\left(S^{1}\right)$, then $\theta$ is a map such that $\theta\left(e_{i}\right)=\theta_{i} \in \Omega^{2,2}\left(\Delta_{1}\right)$, that is,

$$
\begin{gathered}
\theta_{0}\left(x_{0}, x_{1}\right)=a_{0} d x_{0}^{2}+a_{1} d x_{1}^{2}, \\
\theta_{1}\left(y_{0}, y_{1}\right)=b_{0} d y_{0}^{2}+b_{1} d y_{1}^{2}, \\
\theta_{2}\left(z_{0}, z_{1}\right)=c_{0} d z_{0}^{2}+c_{1} d z_{1}^{2} .
\end{gathered}
$$

Any such form $\theta$ is closed. We also have that $\theta$ is exact, in fact $\theta=d \omega$ where $\omega \in$ $\Omega^{2,2}\left(S^{1}\right)$ is given by (note that 2 is invertible in $\mathbb{Q}_{2}$ )

$$
\begin{aligned}
& \omega_{0}\left(x_{0}, x_{1}\right)=\frac{a_{0}}{2} x_{0} d x_{0}-\frac{a_{0}}{2} d x_{0} x_{0}+\frac{a_{1}}{2} x_{1} d x_{1}-\frac{a_{1}}{2} d x_{1} d x_{1}, \\
& \omega_{1}\left(y_{0}, y_{1}\right)=\frac{b_{0}}{2} y_{0} d y_{0}-\frac{b_{0}}{2} d y_{0} y_{0}+\frac{b_{1}}{2} y_{1} d y_{1}-\frac{b_{1}}{2} d y_{1} y_{1}, \\
& \omega_{2}\left(z_{0}, z_{1}\right)=\frac{c_{0}}{2} z_{0} d z_{0}-\frac{c_{0}}{2} d z_{0} z_{0}+\frac{c_{1}}{2} z_{1} d z_{1}-\frac{c_{1}}{2} d z_{1} d z_{1} .
\end{aligned}
$$

Then $H^{2}\left(\Omega^{*, 2}\left(S^{1}\right)\right)=0$. Finally, $H^{i}\left(\Omega^{*, 2}\left(S^{1}\right)\right)=0$ for $i>2$ because $\Omega^{p, 2}\left(S^{1}\right)=0$ for $p>2$.

We conclude this section with the presentation of a more general version of the noncommutative tame de Rham theorem (Theorem 7.1). Let $M$ be a $\mathbb{Q}_{q}$-module and let $\omega=\eta \otimes a \in \Omega^{p, q}(X) \otimes M$, and $\sigma=\theta \otimes b \in C_{p}\left(X ; \mathbb{Q}_{q}\right) \otimes M$. Then the integral of $\omega$ on $\sigma$ is defined by

$$
\int_{\sigma} \omega=I(\eta)(\theta) \cdot a \otimes b=\int_{\theta} \eta \cdot a \otimes b .
$$

Thus integration defines a morphism of modules $\mathbb{Q}_{q}$-modules $I: \Omega^{p, q}(X) \otimes M \rightarrow C^{p}(X$; $M)$. Finally, we apply Künneth's theorem and Theorem 7.1 to obtain the noncommutative tame de Rham theorem for cohomology. 
THEOREM 7.3. Let $X$ be a simplicial set of finite type. Then for $q \geq 1$ and any $\mathbb{Q}_{q^{-}}$ module $M$ there is a natural isomorphism of $\mathbb{Q}_{q}$-modules

$$
H^{i}\left(\Omega^{*, q}(X), M\right) \stackrel{\cong}{\longrightarrow} H^{i}(X ; M)
$$

for all $i \geq 0$. The isomorphism is induced by integration.

Let $f: M_{1} \rightarrow M_{2}$ be a morphism of $\mathbb{Q}_{q}$-modules. Then for each $p \geq 0, f$ induces two morphisms of $\mathbb{Q}_{q}$-modules $f^{*}: H^{i}\left(\Omega^{p, q}(X), M_{1}\right) \rightarrow H^{i}\left(\Omega^{p, q}(X), M_{2}\right)$ and $f^{*}: H^{i}\left(X ; M_{1}\right)$ $\rightarrow H^{i}\left(X ; M_{2}\right)$. The word "natural" in Theorem 7.3 means that if $f: M_{1} \rightarrow M_{2}$ is a morphism of $\mathbb{Q}_{q}$-modules then the diagram

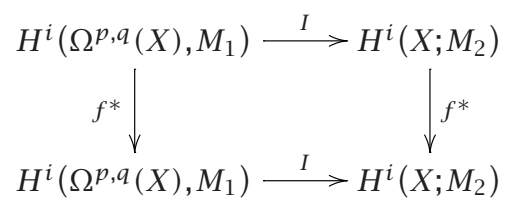

commutes for all $p \geq 0, i \geq 0$.

The existence of an isomorphism $H^{i}\left(\Omega^{*}(X)\right) \rightarrow H^{i}(X ; \Lambda)$, for any commutative ring with a unit $\Lambda$, can be obtained following Cartan's ideas. Such a proof is found in [3, 4]. Cenkl's (1998) noncommutative de Rham theorem is a generalization of a result of Karoubi proved in [12]. In that same paper Karoubi conjectured the noncommutative de Rham theorem using integration being $\Lambda$ a commutative ring containing the rationals.

8. The dual noncommutative complex of Cenkl-Porter. In [20], Scheerer et al. presented a dual version of the tame de Rham theorem. They introduced the chain complex of tame de Rham currents and proved the tame de Rham theorem for homology. In this section, we study the dual of the complex of noncommutative tame forms $\Omega_{*, *}(X)$ on a simplicial set of finite type $X$. We use the facts that $X$ is a simplicial set of finite type and that $\Omega_{n}^{p, q}$ is a finitely generated free $\mathbb{Z}$-module for $p \geq 0$, $q \geq 0, n>0$, and some classical results to prove some basic properties of the complex $\Omega_{*, *}(X)$. Then we prove that for $q \geq 1$ there exists an isomorphism of $\mathbb{Q}_{q}$-modules $H_{i}\left(X ; \mathbb{Q}_{q}\right) \cong H_{i}\left(\Omega_{*, q}(X)\right)$ for all $i \geq 0$.

Let $\Omega^{p, q}\left(\Delta_{n}\right)=\Omega_{n}^{p, q} \otimes_{\mathbb{Z}} \mathbb{Q}_{q}$ be the collection of noncommutative tame $p$-forms of weight $\leq q$ on the $n$-simplex $\Delta_{n}$. Define

$$
\Omega_{p, q}\left(\Delta_{n}\right):=\operatorname{Hom}\left(\Omega^{p, q}\left(\Delta_{n}\right), \mathbb{Q}_{q}\right) .
$$

Elements of $\Omega_{p, q}\left(\Delta_{n}\right)$ are called noncommutative tame de Rham currents.

Consider the maps

$$
\begin{gathered}
\Omega_{p, q}\left(\Delta_{n}\right) \stackrel{\partial}{\longrightarrow} \Omega_{p-1, q}\left(\Delta_{n}\right), \quad \Omega_{p, q}\left(\Delta_{n-1}\right) \stackrel{\delta^{i}}{\longrightarrow} \Omega_{p, q}\left(\Delta_{n}\right), \\
\Omega_{p, q}\left(\Delta_{n+1}\right) \stackrel{s^{i}}{\longrightarrow} \Omega_{p, q}\left(\Delta_{n}\right),
\end{gathered}
$$


where $\partial=\tilde{d}, \delta^{i}=\tilde{\delta}_{i}$, and $s^{i}=\tilde{s}_{i}$ denote the dual of the maps

$$
\begin{array}{ll}
\Omega^{p, q}\left(\Delta_{n}\right) \stackrel{d}{\longrightarrow} \Omega^{p+1, q}\left(\Delta_{n}\right) & \text { (the differential), } \\
\Omega^{p, q}\left(\Delta_{n}\right) \stackrel{\delta_{i}}{\longrightarrow} \Omega^{p, q}\left(\Delta_{n-1}\right) & \text { (the face operators), } \\
\Omega^{p, q}\left(\Delta_{n}\right) \stackrel{s_{i}}{\longrightarrow} \Omega^{p, q}\left(\Delta_{n+1}\right) & \text { (the degeneracy operators). }
\end{array}
$$

Next we use the dual of the contracting homotopy $\jmath_{\lambda}: \Omega^{p+1, q}\left(\Delta_{n}\right) \rightarrow \Omega^{p, q}\left(\Delta_{n}\right)$ (see Lemma 5.7) to prove the Poincaré lemma for noncommutative de Rham currents.

LEMMA 8.1. For every $q \geq 1$,

$$
H_{i}\left(\Omega_{*, q}\left(\Delta_{n}\right)\right)= \begin{cases}\mathbb{Q}_{q}, & \text { for } i=0, \\ 0, & \text { for } i>0 .\end{cases}
$$

Proof. The map $J_{\lambda}: \Omega^{p+1, q}\left(\Delta_{n}\right) \rightarrow \Omega^{p, q}\left(\Delta_{n}\right)$ is a contracting homotopy, that is, $d j_{\lambda}+j_{\lambda} d=1$ (see the proof of Lemma 5.7). Now consider the dual of $\jmath_{\lambda} \tilde{j}_{\lambda}: \Omega_{p, q}\left(\Delta_{n}\right) \rightarrow$ $\Omega_{p+1, q}\left(\Delta_{n}\right)$. For all $w \in \Omega_{p, q}\left(\Delta_{n}\right)$ and for any form $\omega \in \Omega^{p, q}\left(\Delta_{n}\right)$, we have

$$
\begin{aligned}
\partial \tilde{\jmath}_{\lambda}(w)(\omega)+\tilde{\jmath}_{\lambda} \partial(w)(\omega) & =(w)\left(\jmath_{\lambda} d \omega\right)+(w)\left(d \jmath_{\lambda} \omega\right) \\
& =(w)\left(\jmath_{\lambda} d(\omega)+d \jmath_{\lambda}(\omega)\right)=w(\omega),
\end{aligned}
$$

in other words, $\partial \tilde{\jmath}_{\lambda}+\tilde{\jmath}_{\lambda} \partial=1$. Therefore, if $\partial(w)=0$ there exists $v \in \Omega_{p+1, q}\left(\Delta_{n}\right)$ such that $\partial v=w$. Namely, $v=\tilde{\jmath}(w)$.

Using Proposition 5.6 and [14, Theorem III.6.3] we deduce the following proposition.

Proposition 8.2. The sequence

$$
0 \longrightarrow \Omega_{p, q}\left(\partial \Delta_{k}\right) \longrightarrow \Omega_{p, q}\left(\Delta_{k}\right) \longrightarrow \Omega_{p, q}\left(\Delta_{k}, \partial \Delta_{k}\right) \longrightarrow 0
$$

is an exact sequence of $\mathbb{Q}_{q}$-modules for all $p \geq 0$ and $q \geq 1$.

Let $X$ be a simplicial set of finite type. Let $X_{k}$ denote the $k$-skeleton of $X$. Let $C_{*}(X)$ be the complex of unreduced chains of $X$ (as an abelian group) and let

$$
C_{*}\left(X ; \mathbb{Q}_{q}\right):=C_{*}(X) \otimes_{\mathbb{Z}} \mathbb{Q}_{q} .
$$

The noncommutative tame de Rham currents of type $(p, q)$ (of first kind) on $X$ are the elements of

$$
\Omega_{p, q}(X):=\operatorname{Hom}\left(\Omega^{p, q}(X), \mathbb{Q}_{q}\right) .
$$

For $q \geq 1$ define $I: C_{p}\left(X ; \mathbb{Q}_{q}\right) \rightarrow \Omega_{p, q}(X)$ as follows: given $\sigma=\sum_{\ell} \sigma_{\ell} \otimes a_{\ell} \in C_{p}\left(X ; \mathbb{Q}_{q}\right)$ and $\omega \in \Omega^{p, q}(X)$ then

$$
I(\sigma)(\omega)=\int_{\sigma} \omega=\sum_{\ell} a_{\ell} \int_{\sigma_{\ell}} \omega .
$$


Let $\left\{\Delta_{k, j}: j \in J\right\}$ be the set of simplexes of $X_{k}$. Because $X$ is of finite type, we have

$$
\Omega_{*, q}\left(X_{k}, X_{k-1}\right)=\operatorname{Hom}\left(\Omega^{*, q}\left(X_{k}, X_{k-1}\right), \mathbb{Q}_{q}\right)=\bigoplus_{j} \Omega_{*, q}\left(\Delta_{k, j}, \partial \Delta_{k, j}\right) .
$$

Then as a consequence of Proposition 8.2, we have the following result.

Proposition 8.3. The sequence

$$
0 \longrightarrow \Omega_{*, q}\left(X_{k-1}\right) \longrightarrow \Omega_{*, q}\left(X_{k}\right) \longrightarrow \Omega_{*, q}\left(X_{k}, X_{k-1}\right) \longrightarrow 0
$$

is an exact sequence of $\mathbb{Q}_{q}$-modules for all $q \geq 1$.

If $X$ is a simplicial set of finite type, then there exists a natural isomorphism of $\mathbb{Q}_{q}$-modules (by [16, Theorem V.4.1])

$$
C_{p}\left(X_{k} ; \mathbb{Q}_{q}\right) \cong \operatorname{Hom}\left(\operatorname{Hom}\left(C_{p}\left(X_{k}\right), \mathbb{Q}_{q}\right), \mathbb{Q}_{q}\right)=\operatorname{Hom}\left(C^{p}\left(X_{k} ; \mathbb{Q}_{q}\right), \mathbb{Q}_{q}\right)
$$

for all $k \geq 0, p \geq 0$. Let $\tilde{I}: C_{p}\left(\Delta_{k} ; \mathbb{Q}_{q}\right) \rightarrow \Omega_{p, q}\left(\Delta_{k}\right)$ be dual to the morphism $I: \Omega^{p, q}\left(\Delta_{k}\right)$ $\rightarrow C^{p}\left(\Delta_{k} ; \mathbb{Q}_{q}\right)$. Then Proposition 6.7 implies the following result.

Proposition 8.4. The diagram

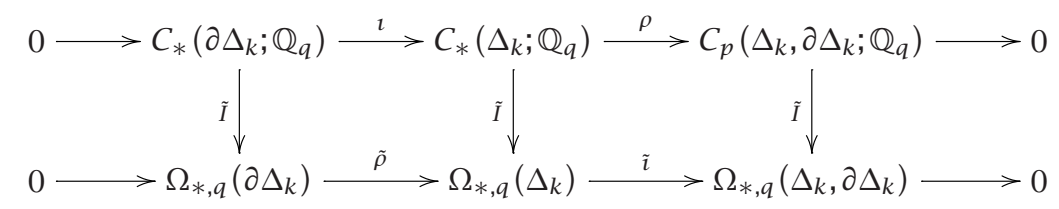

commutes for all $q \geq 1, p \geq 0$ ( 1 and $\rho$ denote inclusion and projection, respectively).

9. The noncommutative tame de Rham theorem for homology. In this section, we prove that the dual map $I$ induces an isomorphism of $\mathbb{Q}_{q}$-modules between the homology of a simplicial set of finite type and the homology of the complex of noncommutative tame de Rham currents.

THEOREM 9.1. Let $X$ be a simplicial set of finite type. Then for $q \geq 1$, the map

$$
\tilde{I}: H_{i}\left(X ; \mathbb{Q}_{q}\right) \stackrel{\cong}{\longrightarrow} H_{i}\left(\Omega_{*, q}(X)\right),
$$

induced by integration, is an isomorphism of $\mathbb{Q}_{q}$-modules for all $i \geq 0$.

Proof. Induction on the skeleta of $X$. For $k=0$, the statement is true because

$$
H_{i}\left(X ; \mathbb{Q}_{q}\right)=\left\{\begin{array}{ll}
\mathbb{Q}_{q}, & \text { if } i=0, \\
0, & \text { if } i>0,
\end{array} \quad H_{i}\left(\Omega_{*, q}(X)\right)= \begin{cases}\mathbb{Q}_{q}, & \text { if } i=0, \\
0, & \text { if } i>0 .\end{cases}\right.
$$

Suppose that the proposition is true for the $\ell$-skeleta $X_{\ell}$, for $\ell<k$. Consider the following commutative diagram:

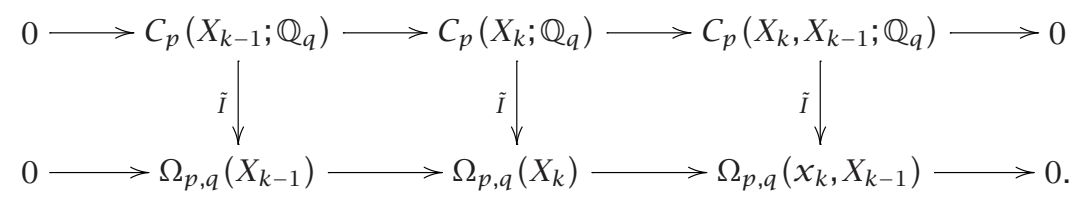


The first row is exact. The second row is exact by Proposition 8.3. Then we have the following commutative diagram, where the rows are exact

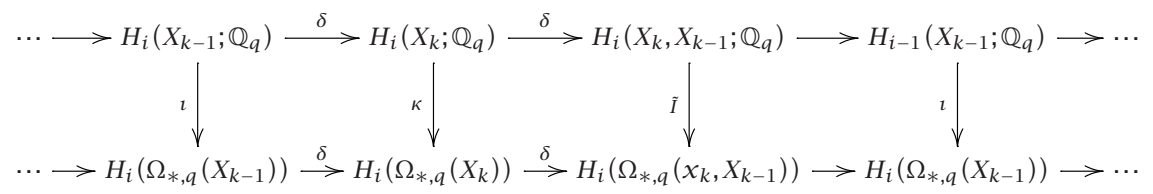

The $\imath$ 's are isomorphisms by induction hypothesis. We prove that $\kappa$ is an isomorphism. Let $\left\{\Delta_{k, j}: j \in J\right\}$ be the $k$-simplices of $X_{k}$. Then the morphisms of $\mathbb{Q}_{q}$-modules

$$
\begin{aligned}
\Omega_{*, q}\left(X_{k}, X_{k-1}\right) & \cong \bigoplus_{j} \Omega_{*, q}\left(\Delta_{k, j}, \partial \Delta_{k, j}\right), \\
C_{*}\left(X_{k}, X_{k-1} ; \mathbb{Q}_{q}\right) & \cong \bigoplus_{j} C_{*}\left(\Delta_{k, J}, \partial \Delta_{k, j} ; \mathbb{Q}_{q}\right)
\end{aligned}
$$

are isomorphism of $\mathbb{Q}_{q}$-modules.

Then it is enough to prove that integration induces an isomorphism

$$
\tilde{I}: H_{i}\left(\Delta_{k}, \partial \Delta_{k} ; \mathbb{Q}_{q}\right) \longrightarrow H_{i}\left(\Omega_{*, q},\left(\Delta_{k}, \partial \Delta_{k}\right)\right) .
$$

By Proposition 8.4 the diagram

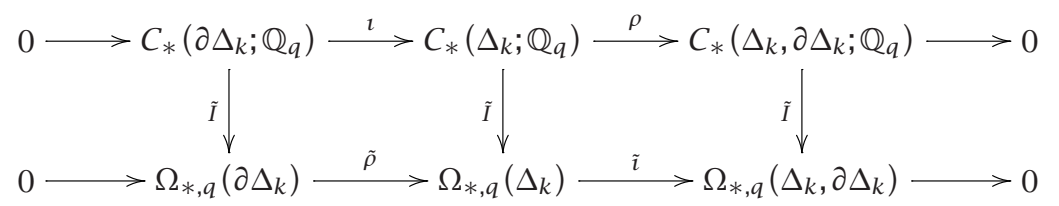

commutes. Then we have the following commutative diagram, with exact rows

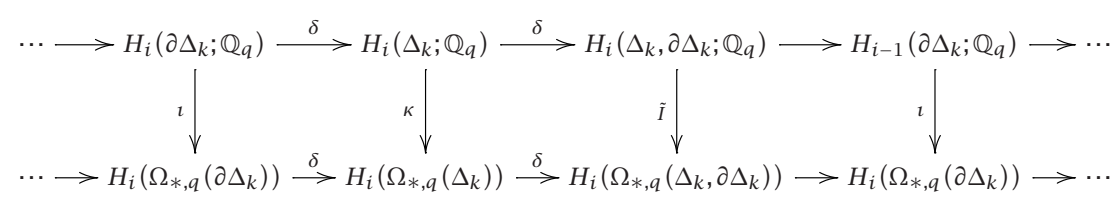

Then we apply five lemma and conclude that $\tilde{I}$ is an isomorphism.

Finally, we apply Künneth's theorem and obtain the noncommutative tame de Rham theorem for homology.

THEOREM 9.2. Let $X$ be a simplicial set of finite type. Then for any $q \geq 1$ and any $\mathbb{Q}_{q}$-module $M$, there is a natural isomorphism of $\mathbb{Q}_{q}$-modules

$$
H_{i}(X ; M) \stackrel{\cong}{\longrightarrow} H_{i}\left(\Omega_{*, q}(X), M\right)
$$

for all $i \geq 0$. The isomorphism is induced by integration. 
The word "natural" in the previous theorem means that for any $\mathbb{Q}_{q}$-modules $M_{1}$ and $M_{2}$ and for any morphism of $\mathbb{Q}_{q}$-modules $f: M_{1} \rightarrow M_{2}$, then the diagram

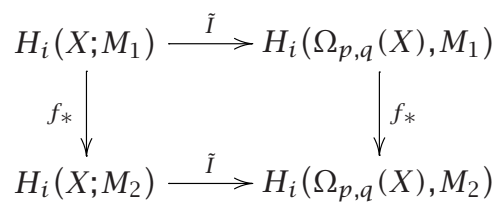

commutes for all $p \geq 0, i \geq 0$, where $f_{*}: H_{i}\left(X ; M_{1}\right) \rightarrow H_{i}\left(X ; M_{2}\right)$ and $f_{*}: H_{i}\left(\Omega_{p, q}(X)\right.$, $\left.M_{1}\right) \rightarrow H_{i}\left(\Omega_{p, q}(X), M_{2}\right)$ are the morphisms of $\mathbb{Q}_{q}$-modules induced by $f$.

10. Cofiltered chain complexes and noncommutative tame de Rham currents. Our study of noncommutative de Rham currents and the noncommutative tame de Rham theorem for homology was originally inspired by the investigations of Scheerer et al. in [20], but our approach differs from theirs. In this section, we introduce the complex of noncommutative tame de Rham currents of second kind on a simplicial set $X, \mathscr{T}_{*, *}(X)$ which is a noncommutative version of the tame de Rham currents of Scheere et al. Then we show that, for all $q \geq 0$ there exists an isomorphism of $\mathbb{Q}_{q^{-}}$ modules $\Omega_{*, *}(X) \cong \mathscr{T}_{*, *}(X)$.

For the complex $F(X)$, such that for all $n$,

$$
F_{n}(X)_{p, q}= \begin{cases}0, & \text { for } p>0, \\ C_{n}(X) \otimes \mathbb{Q}_{q}, & \text { for } p=0 .\end{cases}
$$

Note that $F_{n}(X)_{0, q-1} \otimes \mathbb{Q}_{q}=C_{n}(X) \otimes \mathbb{Q}_{q-1} \otimes \mathbb{Q}_{q} \cong C_{n}(X) \otimes \mathbb{Q}_{q}$. Then we can consider the respective identities as restrictions maps $\rho_{q}: F_{n}(X)_{0, q} \rightarrow F_{n}(X)_{0, q-1}$. Therefore, $F(X)$ is a cofiltered chain complex.

Consider $\Omega_{p, q}\left(\Delta_{n}\right):=\operatorname{Hom}_{\mathbb{Z}}\left(\Omega^{p, q}\left(\Delta_{n}\right), \mathbb{Q}_{q}\right)$ and define the restriction maps $\rho_{q}$ : $\Omega_{p, q}\left(\Delta_{n}\right) \rightarrow \Omega_{p, q-1}\left(\Delta_{n}\right) \otimes \mathbb{Q}_{q}$ as the dual of the inclusions $i_{q}: \Omega^{p, q-1}\left(\Delta_{n}\right) \rightarrow \Omega^{p, q}\left(\Delta_{n}\right)$ (Proposition 5.3). Then $\Omega_{-, *}^{\bullet}=\bigoplus_{p, n \geq 0}\left(\cup_{q \geq 1} \Omega_{p, q}\left(\Delta_{n+1}\right)\right.$ ) is a cosimplicial cofiltered chain complex. It is also a coalgebra; the coproduct is obtained by dualizing the multiplication of noncommutative differential forms

$$
\Omega^{p_{1}, q_{1}}\left(\Delta_{n}\right) \otimes \Omega^{p_{2}, q_{2}}\left(\Delta_{n}\right) \stackrel{\mu}{\longrightarrow} \Omega^{p_{1}+p_{2}, q_{1}+q_{2}}\left(\Delta_{n}\right),
$$

$\mu$ is a noncommutative graded bilinear map of simplicial groups such that for all $0 \leq a_{1} \leq q_{1}, 0 \leq a_{2} \leq q_{2}$

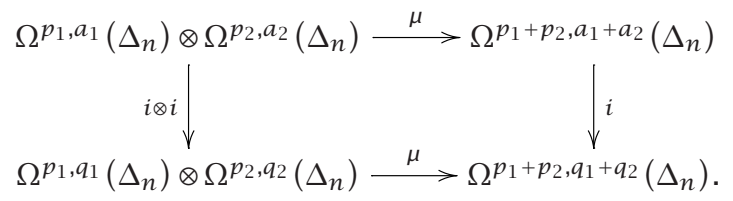

Recall that if $V=\left\{V_{q}, \mathbb{Q}_{q}\right\}$ and $W=\left\{W_{q}, \mathbb{Q}_{q}\right\}$ are two cofiltered chain complexes then the tensor product

$$
(V \otimes W)_{q}=\frac{\lim }{q_{1}+q_{2} \leq q}\left(V_{q_{1}} \otimes W_{q_{2}} \otimes \mathbb{Q}_{q}\right)
$$

is a cofiltered chain complex. 
Consider the cofiltered chain complex $\mathscr{T}(X)$ of noncommutative tame de Rham currents of second kind on $X$, where $\mathscr{T}_{p, q}(X)$ is defined as the quotient of

$$
\bigoplus_{n \geq 0}\left(F_{n}(X) \otimes \Omega^{n}\right)_{p, q}
$$

by the subspaces generated by the images of the maps

$$
\left(\alpha_{*} \otimes 1-1 \otimes \alpha^{*}\right): F_{m}(X) \otimes \Omega^{k} \longrightarrow \bigoplus_{n \geq 0}\left(F_{n}(X) \otimes \Omega^{n}\right)_{p, q}
$$

induced by all the morphisms $\alpha: \Delta(m) \rightarrow \Delta(k)$ in the category of simplicial sets $\Delta$. For all $n$ there exists a natural isomorphism

$$
\left(F_{n}(X) \otimes \Omega^{n}\right)_{p, q} \cong C_{n}(X) \otimes \Omega_{p, q}^{n} .
$$

Then $F(X)_{p, q}$ has a structure of simplicial cofiltered coalgebra.

The de Rham theorem for the complex $\mathscr{T}(X)$ follows from Theorem 9.2 and from the following proposition.

Proposition 10.1. Let $X$ be a simplicial set of finite type. Then for all $q \geq 0$ there is a natural isomorphism of $\mathbb{Q}_{q}$-modules

$$
\Omega_{*, q}(X) \cong \mathscr{T}_{*, q}(X) \quad \forall q \geq 1
$$

Proof. First, we prove that the cochain complex $\Omega^{*, q}(X)$ is a naturally isomorphic to the cochain complex $\operatorname{Hom}\left(\mathscr{T}_{*, q}(X), \mathbb{Q}_{q}\right)$. Let $\omega: X_{*} \rightarrow \Omega^{p, q}\left(\Delta_{*}\right)$ be a simplicial map ( $\omega$ is a form on $X$ ). Let $\sigma \in X_{n}$ be an $n$-simplex and $w \in \Omega_{p, q}\left(\Delta_{n}\right):=$ $\operatorname{Hom}\left(\Omega^{p, q}\left(\Delta_{n}\right), \mathbb{Q}_{q}\right)$. Let $[\sigma \otimes w] \in \mathscr{T}_{p, q}(X)$ be the equivalence class containing $\sigma \otimes \omega$ (a current of second kind). Define a homomorphism $\zeta(\omega): \mathscr{T}_{p, q}(X) \rightarrow \mathbb{Q}_{q}$ by

$$
\zeta(\omega)([\sigma \otimes \omega])=w(\omega(\sigma))
$$

We prove that $\zeta(\omega)$ is well defined: suppose that $\theta \otimes \nu \in[\sigma \otimes \omega]$. Then for any morphism $\alpha: \Delta(k) \rightarrow \Delta(m)$ in the category $\Delta$ there are elements $\eta \in X_{m}$ and $z \in \Omega^{k}$ such that

$$
\theta \otimes v=\sigma \otimes w+\left(\alpha_{*} \otimes 1-1 \otimes \alpha^{*}\right)(\eta \otimes z)=\sigma \otimes w+\alpha_{*}(\eta) \otimes z-\eta \otimes \alpha^{*}(z) .
$$

Then

$$
\begin{aligned}
\zeta(\omega)([\theta \otimes v]) & =w(\omega(\sigma))+z\left(\omega\left(\alpha^{*}(\eta)\right)\right)-\alpha_{*}(z)(\omega(\eta)) \\
& =w(\omega(\sigma))+z\left(\alpha^{*}(\omega(\eta))\right)-\alpha_{*}(z)(\omega(\eta)) \\
& =w(\omega(\sigma))+\alpha_{*}(z)(\omega(\eta))-\alpha_{*}(z)(\omega(\eta)) \\
& =w(\omega(\sigma)) \\
& =\zeta(\omega)([\sigma \otimes \omega]) .
\end{aligned}
$$


Thus $\zeta: \Omega^{p, q}(X) \rightarrow \operatorname{Hom}\left(\mathscr{T}_{p, q}(X), \mathbb{Q}_{q}\right)$ is a homomorphism. Note that

$$
\zeta(d \omega)([\sigma \otimes \omega])=w(d \omega(\sigma))=\partial(w(\omega(\sigma)))=\partial \zeta(\omega)([\sigma \otimes \omega]) .
$$

Now we prove that $\zeta$ is injective. Suppose that $\omega, \eta \in \Omega^{p, q}(X)$ with $\zeta(\omega)=\zeta(\eta)$. Then for all $n$-simplex $\sigma \in X_{n}$ and $w \in \Omega_{p, q}\left(\Delta_{n}\right)$ we have $\zeta(\omega)([\sigma \otimes w])=\zeta(\eta)([\sigma \otimes$ $w])$. This is equivalent to $w(\omega(\sigma))=w(\eta(\sigma))$ for all $\sigma$. Then $\omega(\sigma)=\eta(\sigma)$ for all $\sigma$. Hence $\omega=\eta$.

Now we prove that $\zeta$ is surjective. Let $\bar{\omega}: \mathscr{T}_{*, q}(X) \rightarrow \mathbb{Q}_{q}$ be a morphism of $\mathbb{Q}_{q^{-}}$ modules. Let $\sigma \in X_{n}$ be an $n$-simplex. Consider the homomorphism $\bar{\omega}(\sigma): \Omega_{*, q}\left(\Delta_{n}\right) \rightarrow$ $\mathbb{Q}_{q}$ defined by

$$
\bar{\omega}(\sigma)(w)=\bar{\omega}([\sigma \otimes w]) .
$$

Thus $\bar{\omega}(\sigma) \in \operatorname{Hom}\left(\Omega_{*, q}\left(\Delta_{n}\right), \mathbb{Q}_{q}\right)$. Then there exists a unique form $\omega(\sigma) \in \Omega^{*, q}\left(\Delta_{n}\right)$ such that

$$
\bar{\omega}(\sigma)(w)=w(\omega(\sigma))
$$

$\varphi(\omega(\sigma))=\bar{\omega}(\sigma)$, where $\varphi$ is the natural isomorphism between a module and its dual (see [16, Theorem V.4.1]). Let $\alpha: \Delta(n) \rightarrow \Delta(k)$ be a morphism in the category $\Delta$. Then $\left[\alpha_{*} \sigma \otimes z\right]=\left[\sigma \otimes \alpha^{*} z\right]$ for all $z \in \Omega_{p, q}\left(\Delta_{k}\right)$. Then

$$
z\left(\omega\left(\alpha_{*} \sigma\right)\right)=\alpha^{*} z(\omega(\sigma))=z\left(\alpha^{*}(\omega(\sigma))\right) .
$$

Thus the map $\sigma \mapsto \omega(\sigma)$ is a simplicial map $\omega: X_{*} \rightarrow \Omega^{p, q}\left(\Delta_{*}\right)$. Moreover,

$$
\zeta(\omega)([\sigma \otimes w])=w(\omega(\sigma))=\bar{\omega}(\sigma)(w)=\bar{\omega}([\sigma \otimes w]),
$$

and $\zeta(\omega)=\bar{\omega}$.

Finally we apply [16, Theorem V.4.1] to conclude the proof.

ACKNOWLEDGments. This paper is essentially a part of the author's thesis written under supervision of Professor Bohumil Cenkl (Northeastern University), whom I gratefully thank for advice and encouragement. The author was supported by University of the Andes (Mérida and Trujillo, Venezuela) and the National Council for Scientific and Technological Researches (Caracas, Venezuela).

\section{REFERENCES}

[1] P. Boullay, F. Kiefer, M. Majewski, M. Stelzer, H. Scheerer, M. Unsöld, and E. Vogt, Tame Homotopy Theory via Differential Forms, Freie Universität, Berlin, preprint No. 233, 1986.

[2] H. Cartan, Théories cohomologiques, Invent. Math. 35 (1976), 261-271.

[3] B. Cenkl, Noncommutative differential forms and cohomology operations, preprint, 1998.

[4] , Noncommutative Geometry, Northeastern University, Boston, 1998, Course Notes.

[5] B. Cenkl, G. Hector, and M. Saralegi, Cohomologie d'intersection modérée. Un théorème de de Rham, Pacific J. Math. 169 (1995), no. 2, 235-289 (French).

[6] B. Cenkl and R. Porter, Differential forms and torsion in the fundamental group, Adv. in Math. 48 (1983), no. 2, 189-204. 
[7] _ de Rham theorem with cubical forms, Pacific J. Math. 112 (1984), no. 1, 35-48.

[8] A. Connes, Noncommutative differential geometry, Inst. Hautes Études Sci. Publ. Math. (1985), no. 62, 257-360.

[9]_ Noncommutative Geometry, Academic Press, California, 1994.

[10] P. G. Goerss, Simplicial chains over a field and p-local homotopy theory, Math. Z. 220 (1995), no. 4, 523-544.

[11] M. Karoubi, Homologie cyclique et K-théorie, Astérisque 149 (1987), 1-147 (French).

[12] __ Formes différentielles non commutatives et cohomologie à coefficients arbitraires [Noncommutative differential forms and cohomology with arbitrary coefficients], Trans. Amer. Math. Soc. 347 (1995), no. 11, 4277-4299 (French).

[13] I. Kris, p-adic homotopy theory, Topology and Its Applications 52 (1993), 279-308.

[14] S. Lang, Algebra, Addison-Wesley, Massachusetts, 1993.

[15] D. Lehmann, Théorie homotopique des formes différentielles (d'aprés D. Sullivan), Astérisque (1990), no. 45, 1-145 (French).

[16] S. Mac Lane, Categories for the Working Mathematician, 2nd ed., Springer-Verlag, New York, 1998.

[17] J. Madore, An Introduction to Noncommutative Differential Geometry and Its Physical Applications, 2nd ed., London Mathematical Society Lecture Note Series, Cambridge University Press, Cambridge, 1999.

[18] J. P. Miller, Simplicial Objects in Algebraic Topology, Chicago Lectures in Mathematics, vol. 11, D. Van Nostrand, London, 1967.

[19] C. Mouet, q-cohomologie non commutative, C. R. Acad. Sci. Paris Sér. I Math. 323 (1996), no. 8, 849-851.

[20] H. Scheerer, K. Schuch, and E. Vogt, Tame Homotopy Theory via de Rham Currents, Freie Universität Berlin, Berlin, preprint No. A91-20, 1991.

[21] E. H. Spanier, Algebraic Topology, McGraw-Hill Book, New York, 1966.

[22] M. Sullivan, A Comprehensive Introduction to Differential Geometry. Vol. I, 3rd ed., Publish or Perish, Houston, 1999.

[23] F. W. Whitney, Foundations of Differentiable Manifolds and Lie Groups, Springer-Verlag, New York, 1983.

Luis Fernando Mejias: Department of Mathematics, Northeastern University, 567 LAKE HALL, BOSTON, MA 02115, USA

E-mail address: 1mejias@ynx.neu.edu 


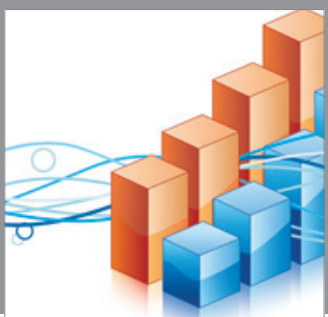

Advances in

Operations Research

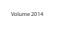

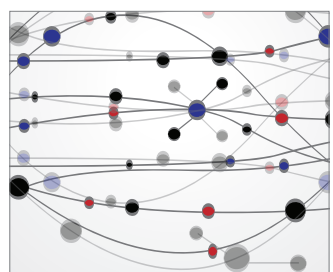

\section{The Scientific} World Journal
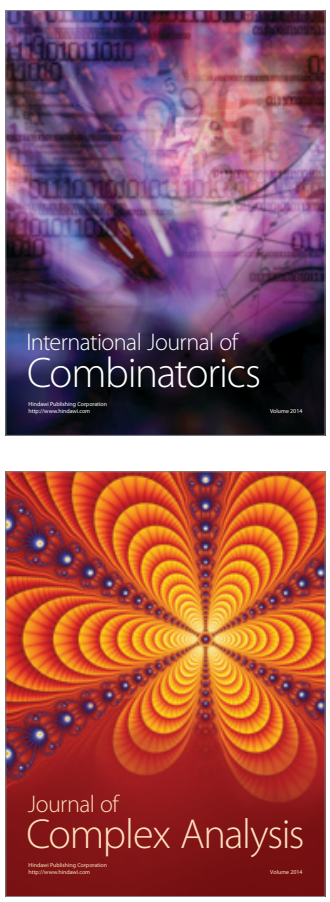

International Journal of

Mathematics and

Mathematical

Sciences
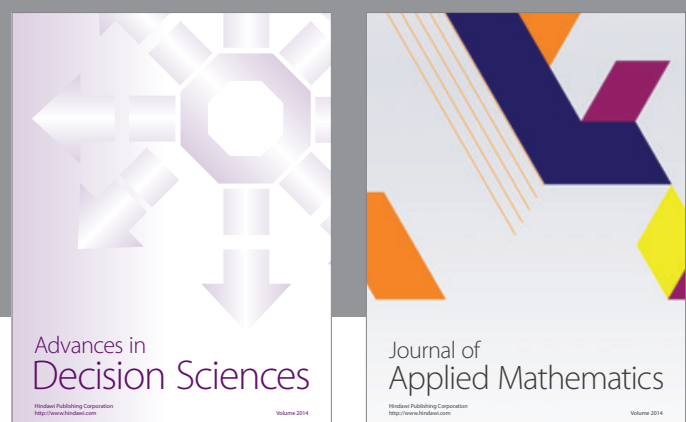

Journal of

Applied Mathematics
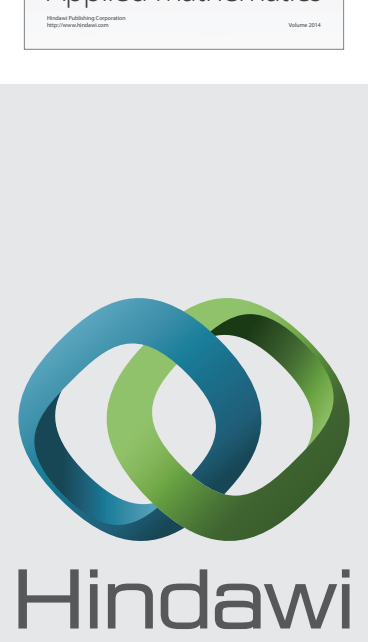

Submit your manuscripts at http://www.hindawi.com
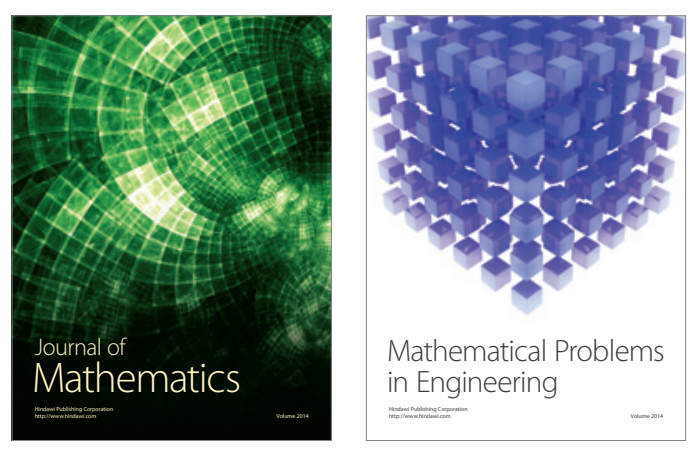

Mathematical Problems in Engineering
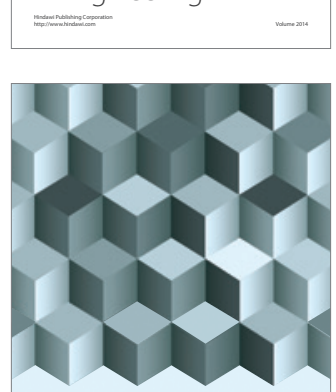

Journal of

Function Spaces
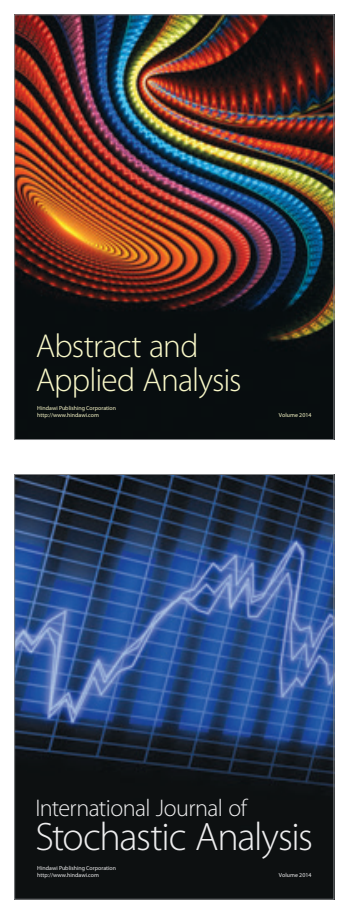

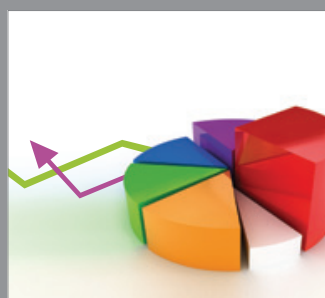

ournal of

Probability and Statistics

Promensencen
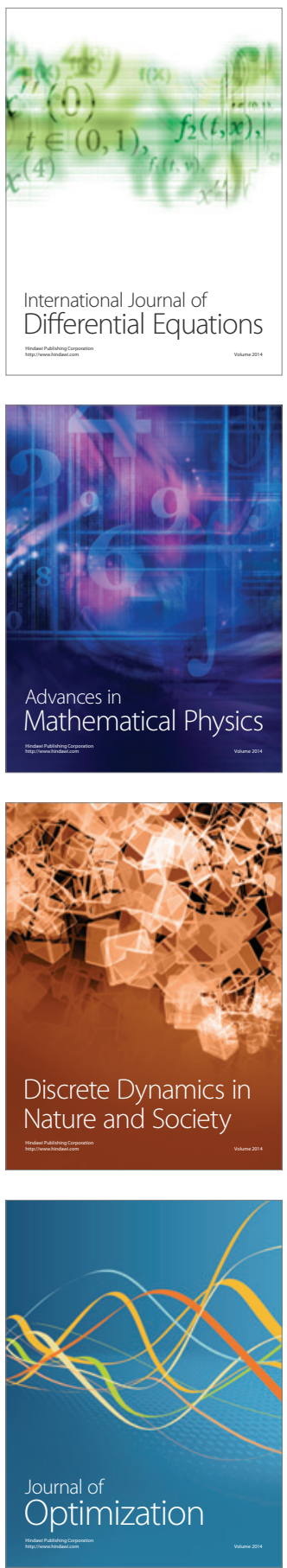